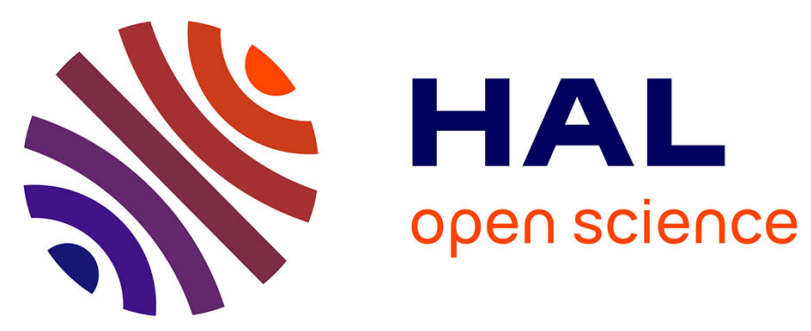

\title{
Investigating the dependence of the temperature of high-intensity discharge (HID) lamp electrodes on the operating frequency by pyrometric measurements
}

J Reinelt, M Westermeier, C Ruhrmann, A Bergner, P Awakowicz, J Mentel

\section{- To cite this version:}

J Reinelt, M Westermeier, C Ruhrmann, A Bergner, P Awakowicz, et al.. Investigating the dependence of the temperature of high-intensity discharge (HID) lamp electrodes on the operating frequency by pyrometric measurements. Journal of Physics D: Applied Physics, 2011, 44 (9), pp.95204. 10.1088/0022-3727/44/9/095204 . hal-00599244

HAL Id: hal-00599244

https://hal.science/hal-00599244

Submitted on 9 Jun 2011

HAL is a multi-disciplinary open access archive for the deposit and dissemination of scientific research documents, whether they are published or not. The documents may come from teaching and research institutions in France or abroad, or from public or private research centers.
L'archive ouverte pluridisciplinaire HAL, est destinée au dépôt et à la diffusion de documents scientifiques de niveau recherche, publiés ou non, émanant des établissements d'enseignement et de recherche français ou étrangers, des laboratoires publics ou privés. 


\title{
Investigating the dependence of the temperature of HID lamp electrodes on the operating frequency by pyrometric measurements
}

\author{
J Reinelt $\dagger$, M Westermeier, C Ruhrmann, A Bergner, $\mathbf{P}$ \\ Awakowicz and J Mentel \\ Ruhr University of Bochum, Electrical Engineering and Plasma Technology, D-44780 \\ Bochum, Germany \\ E-mail: juergen.mentel@ruhr-uni-bochum.de
}

\begin{abstract}
Phase resolved temperature distributions are determined along a rod shaped tungsten electrode, by which an ac arc is operated within a model lamp filled with argon. Switched dc and sinusoidal currents are applied with amplitudes of several amps and operating frequencies being varied between $10 \mathrm{~Hz}$ and $10 \mathrm{kHz}$. The temperature is deduced from the grey body radiation of the electrode being recorded with a spectroscopic measuring system. Phase resolved values of the electrode tip temperature $T_{\text {tip }}$ and of the power input $P_{i n}$ are determined comparing the measured temperature distributions with the integral of the one dimensional heat balance with these parameters as integration constants. They are supplemented by phase resolved measurements of the sum of cathode and anode fall called electrode sheath voltage. If a switched dc current is applied it is found that both quantities are within the cathodic phase only marginally higher than for a cathode being operated with a dc current. $T_{\text {tip }}$ and $P_{\text {in }}$ start to decrease for low currents and to increase for high currents at the beginning of the anodic phase. But with increasing operating frequency the deviations from the cathodic phase are reduced until they cannot be resolved for frequencies of several $\mathrm{kHz}$. A more pronounced modulation but the same tendencies are observed with a sinusoidal current waveform. For $10 \mathrm{kHz}$ a diffuse arc attachment with an almost phase independent electrode tip temperature, which deviates only marginally from that of a dc cathode, and an electrode sheath voltage proportional to the arc current is established with both current waveforms.
\end{abstract}

Submitted to: J. Phys. D: Appl. Phys. 


\section{Introduction}

A quantitative description has been achieved now of the dc operation of cathodes in high intensity discharge (HID) lamps at least in the case of plain lamp fillings, e.g. with noble gases or mercury. It is on one hand an outcome of intense theoretical studies and on the other hand of experimental investigations, mainly at a model lamp, within the last years $[1,2,3,4,5,6,7,8,9,10,11,12,13,14,15]$. The description of the dc operation of anodes in HID lamps is less advanced, but at least a qualitative understanding has been achieved for cold and to some extent also for hot HID anodes [16, 17, 18, 19]. The different properties of the cathodic and anodic arc attachment in HID lamps can be distinctly defined now. The cathodic arc attachment is determined by a thin plasma boundary layer covering the electrode surface. It is characterized by a much higher electrical power input per unit volume than into the bulk plasma and a power flux to the cathode of high density. It heats the cathode so that thermionic electron emission can take place. The layer decouples the cathode from the bulk plasma. Accordingly, its properties are completely determined by the cathode fall $U_{c}$ and the temperature of the cathode surface $T_{c}[10,11]$. The power balance of the system plasma boundary layer - cathode has different solutions beneath a distinct arc current corresponding to the experimentally observed diffuse and spot mode of cathodic arc attachment. The critical current is determined by the electrode geometry and the gas filling. In the case of the anodic arc attachment a boundary layer with some extension perpendicular to the electrode surface is formed. It consists of a constriction zone and a thin sheath immediately in front of the anode. The constriction zone produces a positive voltage drop in front of the anode; the voltage drop across the sheath may be negative especially in the case of a cold anode when a thermionic electron emission of the anode can be neglected. The resulting anode fall $U_{a}$ is positive, but its influence onto the power input into the electrode is, different to that of the cathode fall $U_{c}$, marginal $[19,20]$. With increasing anode temperature the thermionic electron emission exerts a rising influence onto the anodic arc attachment. The emitted electrons return to the anode with an enhanced kinetic energy withdrawn from the plasma boundary layer in front of it. Different to the cathode the thermionic electron emission of the anode cools the anodic plasma boundary layer and heats the anode. It causes a spreading of the anodic plasma boundary layer across the anode surface. Comparing cathode and anode it was found, that at low currents the power input into the cathode and with it the cathode temperature is higher than the power input and tip temperature of the anode. But at a definite current, which is determined by the properties of the electrode and of the gas atmosphere, the power input into the anode and with it the anode tip temperature exceed those of the cathode $[16,19]$.

However, it was shown recently operating a model lamp with a sinusoidal or switched dc current $[21,22]$ that the results obtained in the case of dc operation of HID electrodes can not simply be transferred to the cathodic and anodic phase in the case of ac operation. Interferences are observed between the cathodic and anodic arc attachment, 
increasing with rising operating frequency. They are reflected by an adaptation of the time/phase dependent variation of the electrode sheath voltage (ESV), representing the sum of the cathode fall $U_{c}$ and the anode fall $U_{a}$, to that of the current waveform proceeding with increasing operating frequency. Similar results were found if the ESVs of high pressure mercury lamps operated with sinusoidal currents of different frequencies were deduced from voltage measurements at lamps of different length but otherwise identical properties. Moreover it was indicated by these measurements that the anode fall $U_{a}$ may become intermittently negative in the case of an operation with a sinusoidal current $[23,24]$. Besides the spot mode of cathodic arc attachment disappears with increasing frequency. It is only formed if special structures are present on the electrode surface [22]. Pronounced changes are also observed if the electrical power supply of metal halide HID lamps operated in quartz or transparent YAG tubes is changed from a dc current to a sinusoidal or switched dc current [25, 26, 27, 28, 29].

The theoretical treatment of ac operated HID lamp electrodes is still at the beginning. The reason is that up to now an appropriate model of the anode boundary layer is missing. In [30] the problem was circumvented by modeling only the cathode of a high pressure mercury lamp being operated with a rectified sinusoidal current. Mode changes from the diffuse into the spot mode within the up swinging branch and from the spot into the diffuse mode within the declining branch of the sinusoidal half wave could be simulated for an operating frequency $f=50 \mathrm{~Hz}$, low currents and appropriate electrode parameters. For $f=500 \mathrm{~Hz}$ only a diffuse mode of arc attachment could be realized by simulation. Additionally the simulation shows an adjustment of the time variation of the cathode fall $U_{c}(t)$ to the current wave form $i(t)=I_{0}|\sin \omega t|$ when the operating frequency was increased. Finally it was found that for the same $i_{r m s}$ the power input into the cathode becomes lower for the rectified sine wave than in the case of a dc operation. The theoretical predictions were confirmed at least qualitatively by experimental investigations on the cathode of an ac high-pressure mercury discharge [31]. In [26] similar results were obtained in the case of an operation of a high pressure Hg lamp with a $f=120 \mathrm{~Hz}$ switched dc current, if the model given in [30] is extended by a simple anode model being represented by a constant anode heating voltage. The stability of the diffuse mode of cathodic arc attachment is investigated theoretically very detailed in [32] for electrodes being operated with an ac current. The results are in accordance with the experimental finding that the disposition for the formation of a spot like cathodic arc attachment decreases with increasing operating frequency. But also in [32] a very simple anode model is used.

The changes of arc attachment at the electrodes for an increased operating frequency from $10 \mathrm{~Hz}$ to $10 \mathrm{kHz}$ will be investigated in more detail by pyrometric, electric and spectroscopic measurements at the Bochum model lamp [5]. Phase resolved measurements of the temperature distribution along the electrode rod made of pure tungsten are performed in the model lamp filled with argon and supplemented by phase resolved measurements of the ESV. Two different current waveforms are applied: a 
switched dc current and a sinusoidal current. The electrode tip temperature and the power input into the electrode are determined in dependence on the phase angle for operating frequencies from $10 \mathrm{~Hz}$ to $10 \mathrm{kHz}$ for different current amplitudes. They are obtained by comparing the temperature distributions measured along the electrode rod with an integral of the time dependent power balance of the electrode. Finally an interpretation of the results will be given considering the interaction between the electrode and the plasma boundary in front of it. The major part of the results is already given in the $\mathrm{PhD}$ thesis of Reinelt [33].

The power input into the electrode during the cathodic phase and the ESV will be used in another paper to determine electrode falls in the case of ac operation.

\section{Experimental setup}

\subsection{Model lamp}

The Bochum model lamp was already described in several papers; additional sectional drawings are presented in $[5,19]$. Therefore only a sketch will be given here.

The discharge tube of the model lamp is made of fused silica. Its inner diameter amounts to $9 \mathrm{~mm}$. It is mounted in metallic sockets which have several functions. They serve as a vacuum tight feed through for the electrode holders. Additionally they seal the discharge vessel at both ends and manage the gas supply. The lamp tube can be evacuated by a vacuum pumping station to $10^{-4} \mathrm{~Pa}$ before it is filled with noble gases of high purity. The electrode holders are isolated from the whole system to be used as electrical connectors. Furthermore the holders can be moved inside the tube very precisely by stepping motors. This gives the possibility to vary the electrode position and thus the arc length even during operation of the lamp. Tungsten electrodes made of AKS material (tungsten doped with $\mathrm{Al}, \mathrm{K}$ and $\mathrm{Si}$ to achieve a fine grained crystal structure) with a length $l_{E}$ between 10 and $20 \mathrm{~mm}$ and diameter $d_{E}$ between 0.5 and $2 \mathrm{~mm}$ are soldered into the tips of the holders. The electrodes are cooled by silicon oil which is flowing around that part of the electrodes which extends into the electrode holders. By the cooling the electrode temperature at the soldering point is fixed to a constant value. The lamp and the electrodes in it are operated in vertical position. Measurements are mostly performed with the electrode at the lower end of the lamp.

To optimize the spatial resolution of the pyrometric measurements the tube of the model lamp is provided with a quartz window of high optical quality. It is attached to a funnel shaped appendix extending perpendicularly to the tube axis. The attachment surrounds a short longitudinal cut into the wall of the cylindrical tube. Opposite the window a further tube perpendicularly to the discharge tube is added. Serving as a sink for the radiation emitted from the back side of the bright electrode the stray light level is reduced.

The model lamp is operated with a current source (Feucht Elektronik DCU/I 2250-30) being controlled by the output voltage of a signal generator. It is able to provide dc, ac, 
pulsed or transit currents of arbitrary waveform up to a frequency of $30 \mathrm{kHz}$, a current amplitude of $28 \mathrm{~A}$ with a maximum voltage of $400 \mathrm{~V}$. Two different kinds of current waveforms are used: a sinusoidal and a switched dc current.

\subsection{Measurement of the electrode sheath voltage (ESV)}

Quite simple but reliable phase resolved measurements of the ESV can be made at the model lamp using the possibility of a controlled relocation of the electrodes during lamp operation [21].

The arc voltage $U_{\text {arc }}$ can be separated into a cathode fall $U_{c}$, an anode fall $U_{a}$ and a voltage drop along the arc column. The arc column is cylindrical within the model lamp and therefore the axial electrical field strength $E_{\text {arc }}=$ const within the column. This means that $U_{\text {arc }}$ is linearly dependent on the length of the arc given by the distance of the electrode tips $l_{\text {arc }}$.

$$
U_{\text {arc }}=U_{c}+U_{a}+E_{\text {arc }} l_{\text {arc }} .
$$

Therefore it is quite easy to determine the electrode sheath voltage $\operatorname{ESV}(\varphi)=$ $U_{c}(\varphi)+U_{a}(\varphi)$ by a measurement of $U_{\text {arc }}(\varphi)$ in dependence on the arc length $l_{\text {arc }}$ and an extrapolation of $U_{\text {arc }}\left(\varphi, l_{\text {arc }}\right)$ to $l_{\text {arc }}=0$.

The ESV measurements can be done with a phase resolution which is much higher than that of the pyrometric measurements. Therefore it may be helpful to consult ESV measurements for a better interpretation of the pyrometric measurements. Comparing both it has to be considered that the variation of the ESV in dependence on $\varphi$ is mainly determined by the variation of the cathode fall with $\varphi$ at least if the lamp is operated with a switched dc current [21]:

$$
\frac{\partial E S V}{\partial \varphi} \approx \frac{\partial U_{c}}{\partial \varphi}
$$

\subsection{Measurement of the electrode temperature}

Temperature distributions along the electrode rods could be measured sufficiently accurate with commercially available pyrometers as long as the model lamp was operated with a dc current. But their temporal and spatial resolution turned out to be insufficient as soon as the model lamp was operated with alternating currents. By this reason it was substituted by a spectroscopic measuring system. The corresponding experimental set up was already described in $[22,27]$ so that a sketch will be sufficient.

The spectrograph in use is a $250 \mathrm{~mm}$ spectrograph from Chromex with an aperture ratio of $f / 4.0$. A grating is used with a line density of $1200 \mathrm{~mm}^{-1}$. The entrance slit of the spectrograph is adjusted to a width of $100 \mu \mathrm{m}$. To record the radiation emitted by the electrode with the spectrograph an optical imaging system is installed in between. A telecentric arrangement of two achromats forms an image of the electrode on the entrance slit of the spectrograph with a magnification of $M=1$. A Dove prism between the two achromats is used to adjust the image of the electrode parallel to the vertical entrance 
slit of the spectrograph. Thus alignment imperfections from soldering or clamping of the electrode can be corrected.

The output of the spectrograph is recorded with a CCD camera SENSICAM QE from PCO being mounted in the imaging plane of it. The resolution of the camera along the entrance slit is 1376 pixel and along the wavelength axis 1040 pixel. The pixel size amounts to $6.45 \mu \mathrm{m} \times 6.45 \mu \mathrm{m}$. Taking the total magnification of the imaging system into account the maximum length of the electrode which can be detected along the entrance slit of the spectrograph is $\approx 6.78 \mathrm{~mm}$. The camera offers the possibility of an internal binning summing up the counts of a given number of adjacent pixel. The dynamic range of the camera is 14 bits which implies a maximum count rate of 4096 counts per pixel. The exposure time of the camera $t_{\text {exp }}$ can be varied between $500 \mathrm{~ns}$ and $3600 \mathrm{~s}$. The triggering delay time of the camera is very short compared to $t_{\text {exp }}=500 \mathrm{~ns}$. The CCD chip of the camera is sensitive within a wavelength interval from 280 to $1100 \mathrm{~nm}$. A calibration of the optical system in absolute values of radiance is needed for a measurement of the electrode temperature. For this purpose a tungsten ribbon lamp (Wi17g3 from OSRAM) with a known radiance in dependence on wavelength for a specified current is positioned at the same place as the lamp under investigation.

The temporal resolution of the camera is limited by the read out time of the CCD chip. It amounts to approximately $90 \mathrm{~ms}$. This means that at higher operation frequencies $(\mathrm{f} \geq 5 \mathrm{~Hz}$ ) not more than one measurement within one period can be made. Therefore only phase resolved measurements are is possible. The substitution of a temporal by a phase resolution does not raise a problem as long as the lamp operation is really periodic. In this case only a well defined periodical trigger signal is required as a starting point for a series of successively phase delayed exposures.

The "sync" output of the signal generator by which the current source is controlled may provide an appropriate trigger pulse; but the delay time caused by the amplifier varies too much in dependence on the operating parameters. Thus the reference phase has to be generated on the output side of the amplifier. The current zero crossing is inapplicable since the current may not rise fast enough after zero crossing to be able to generate an exact trigger pulse. Therefore the trigger signal is deduced from the voltage zero crossing of the lamp [21]. To trigger the camera only the rising edge of the voltage signal is used. The uncertainty of the trigger pulse can be denoted with a maximum of $2 \mu \mathrm{s}$. To shift the trigger signal from voltage zero crossing to take pictures at different phase angles a delay generator (STANFORD RESEARCH SYSTEM DG 535) is used. A block diagram of the trigger set up is already given in [22].

It is favorable to measure the radiance at a wavelength in the infrared spectral region for temperature measurements at a solid surface. Thus a segment of the spectrum emitted by the electrode is used which is as far as possible in the infrared region, but still within the sensitivity range of the CCD camera. A second condition is that plasma radiation can be neglected compared to the emission of the electrode surface within the recorded spectral interval. A suitable wavelength for these measurements will be $\lambda_{\text {meas }}=718 \mathrm{~nm}$. 
The plasma radiation emitted by the model lamp in this wavelength region is very weak. An edge filter with an edge wavelength $\lambda_{\text {edge }}=600 \mathrm{~nm}$ is used to suppress all radiation at wavelength below $\lambda_{\text {edge }}$. It prevents a distortion of the measurement by the second order spectrum produced by the grating at $\lambda_{\text {meas }} / 2$. The transmission $\operatorname{Tr}$ of the quartz window of the model lamp, which has to be taken into account, is mainly determined by the reflection at the front side and at the back side of the plate. It amounts to $\operatorname{Tr} \leq 93.25 \%$ at $\lambda_{\text {meas }}=718 \mathrm{~nm}$.

A spatial profile of the spectral radiance of the electrode at $\lambda=718 \mathrm{~nm}$ is recorded by a single measurement at a selected phase angle $\varphi$. A grey body radiator is assumed to determine spatial temperature profiles from the measured radiances. The spectral radiance of the grey body $I_{g b}(\lambda, T)$ is given by Planck's law and a temperature and wavelength dependent spectral emissivity $\varepsilon(\lambda, T)<1$.

$$
I_{g b}(\lambda, T)=\varepsilon(\lambda, T) \frac{2 h c_{0}^{2}}{\lambda^{5}}\left(\exp \left(\frac{h c_{0}}{\lambda k_{B} T}\right)-1\right)^{-1}
$$

For tungsten, values of $\varepsilon(\lambda, T)$ are published in literature. Comprehensive investigations showed that the values of deVos [34] are the most trustable ones. Comparing $I_{g b}(\lambda=$ $718 \mathrm{~nm}, T)$ calculated in dependence on $T$ with measured values $I_{g b}(z)$ spatial profiles $T(z, \varphi)$ are determined.

The accuracy of the temperature measurements is mainly affected by questionable values of $\varepsilon$ in the vicinity of the electrode tip. On one hand the uncertainty of $\varepsilon(T, \lambda)$, given in literature, rises if the temperature approaches the melting point of tungsten, on the other hand the surface roughness and with it an enhancement of $\varepsilon$ becomes more pronounced in the vicinity of the electrode tip. Reliable values of the electrode tip temperature $T_{t i p}$ were obtained in [5] for electrodes operated with a dc current by matching measured temperature distributions $T(z)$ within a region of confidence between a lower temperature limit $T_{l}$ and an upper limit $T_{u p}<T_{\text {tip }}$ by a least square fit procedure to numerical solutions of the one dimensional heat balance of the electrode. The electrode tip temperature $T_{\text {tip }}$ and the total power input into the electrode $P_{\text {in }}$ were determined by extrapolating the solutions up to the electrode tip. It was shown that the tip temperature $T_{\text {tip }}$ obtained with a too low value of $\varepsilon$ may be exaggerated up to $100 \mathrm{~K}$ for a cathode operated with a dc current [5]. The question is how far the error of the electrode temperature measurement and with it the determination of the power input into the electrode can be reduced in the case of an ac operation by a similar approach as in $[5]$.

\section{Time/phase dependent power loss calculation from measured temperature distributions}

A rotational symmetric electrode temperature distribution is assumed as it is realized by a diffuse attachment of an arc on the electrode. Moreover the $r$-dependence of $T$ along the electrode rod is neglected. A thin disk of the electrode of the thickness $d z$ 
and a change of the phase angle $d \varphi$ is considered to calculate the power balance of the electrode in dependence on time/phase. Figure 1 shows the coordinate system for the electrode geometry. The temperature is $T=T_{\text {tip }}$ at the position $z=0$. Since during

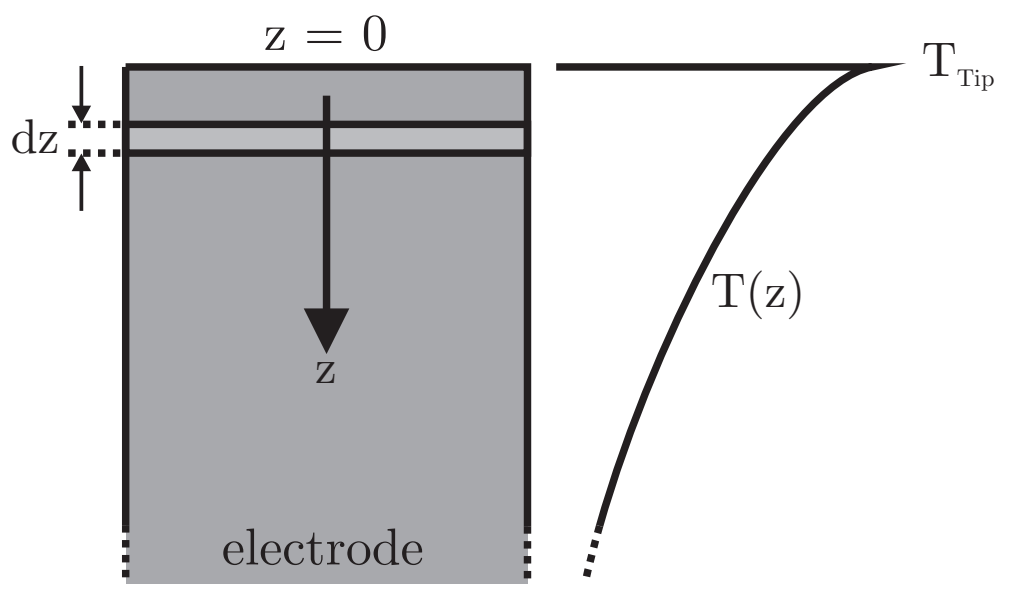

Figure 1. Definition of the coordinate system for the time dependent electrode input power calculation

the exposure time $t_{e}=\varphi_{e} \omega^{-1}$ of the camera the partial derivative $\left(\frac{\partial T}{\partial t_{e}}\right)_{t_{e}}=\omega\left(\frac{\partial T}{\partial \varphi}\right)_{\varphi_{e}}$ is almost constant, the spatial change $\partial T / \partial z$ can be substituted by the total change $d T / d z$. Thus, the heat balance of the electrode disk can be written as:

$$
r_{E}^{2} \pi \frac{d}{d z}\left(\kappa(T) \frac{d T}{d z}\right)=-2 \pi r_{E} q_{E}(T)+2 \pi r_{E} \epsilon_{t o t} \sigma_{S B} T^{4}+r_{E}^{2} \pi \rho_{M} c_{p}(T) \omega\left(\frac{\partial T}{\partial \varphi}\right)_{\varphi_{e}},
$$

where $r_{E}$ is the electrode radius, $\kappa(T)$ the thermal conductivity of tungsten in dependence on temperature [35], $q_{E}(T)$ the power input per unit area from the plasma into the lateral area of the rod, $\epsilon_{t o t}$ is the total emissivity of tungsten [36], $\sigma_{S B}$ the StefanBoltzmann constant, $\rho_{M}$ the mass density of tungsten and $c_{p}(T)$ its heat capacity per unit mass [37]. A comprehensive presentation of the data is given in [38].

Multiplying eq. 4 first with $d z$, afterwards with $\kappa d T / d z$ and integrating from $T$ to $T_{\text {tip }}$ gives:

$$
\begin{aligned}
& \frac{1}{2} r_{E}^{2} \pi\left[\left(\kappa(T) \frac{d T}{d z}\right)_{T_{t i p}}^{2}-\left(\kappa(T) \frac{d T}{d z}\right)_{T}^{2}\right]=-2 \pi r_{E} \int_{T}^{T_{t i p}} q_{E}(T) \kappa(T) d t \\
& +2 \pi r_{E} \int_{T}^{T_{t i p}} \epsilon_{t o t} \sigma_{S B} T^{4} \kappa(T) d T+r_{E}^{2} \pi \omega \int_{T}^{T_{t i p}} \rho_{M} c_{p}(T) \kappa(T)\left(\frac{\partial T}{\partial \varphi}\right)_{\varphi_{e}} d T
\end{aligned}
$$

Now eq. 5 can be resolved for $(d T / d z)_{T}^{-1}$. It has to be taken into account that $d T / d z<0$ according to figure 1 . The decrease of the electrode temperature with increasing values of $z$ is represented by a minus sign at the right hand side of the equation for $d z$ :

$$
d z=
$$




$$
-\frac{\kappa(T) d T}{\left[\left(\kappa(T) \frac{d T}{d z}\right)_{T_{t i p}}^{2}+\frac{4}{r_{E}} \int_{\tau=T}^{\tau=T_{t i p}} q_{E} \kappa d \tau-\frac{4}{r_{E}} \int_{\tau=T}^{\tau=T_{t i p}} \epsilon_{t o t} \sigma_{S B} \tau^{4} \kappa d \tau-2 \omega \int_{\tau=T}^{\tau=T_{t i p}} \rho_{M} c_{p} \kappa\left(\frac{\partial \tau}{\partial \varphi}\right)_{\varphi_{e}} d \tau\right]^{\frac{1}{2}}}
$$

The first and second term within the square root under the fraction bar on the right hand side of eq. 6 need a further interpretation.

The power input per unit area from the plasma into the end face of the electrode $q_{E}\left(T_{t i p}\right)$ is balanced by heat conduction from the surface of the electrode in its bulk and thermal radiation from the surface into free space:

$$
q_{E}\left(T_{t i p}\right)=-\left(\kappa \frac{d T}{d z}\right)_{T_{t i p}}+\epsilon_{t o t} \sigma_{S B} T_{t i p}^{4}=-\left(\kappa \frac{d T}{d z}\right)_{T_{t i p}}+q_{R} ;
$$

with

$$
q_{R}\left(T_{t i p}\right)=\epsilon_{t o t} \sigma_{S B} T_{t i p}^{4} \ll q_{E}\left(T_{t i p}\right) .
$$

It has to be taken into account that $q_{E}(T)$ contributes only in the vicinity of the electrode tip within the attachment area of the arc to the heat balance of the electrode. Observations show that it is limited to $z \leq r_{E} ; T \geq T\left(z=r_{E}\right)=T_{\text {arc }}$

$$
\begin{aligned}
& q_{E}(T)>0 ; T_{\text {arc }} \leq T \leq T_{\text {tip }} \quad \text { for } z \leq r_{E} ; \\
& q_{E}(T)=0 ; T \leq T_{\text {arc }} \quad \text { for } z \geq r_{E} \\
& \int_{\tau=T_{\text {arc }}}^{\tau=T_{\text {tip }}} q_{E} \kappa d \tau=-\int_{z=0}^{r_{E}} q_{E}(z) \kappa\left(\frac{d \tau}{d z}\right) d z \approx-\left(\kappa \frac{d T}{d z}\right)_{T_{\text {tip }}} \int_{0}^{r_{E}} q_{E}(z) d z \\
& =\left[q_{E}\left(T_{\text {tip }}\right)-q_{R}\left(T_{\text {tip }}\right)\right] \int_{0}^{r_{E}} q_{E}(z) d z .
\end{aligned}
$$

with

$$
-\left(\kappa \frac{d T}{d z}\right)_{T_{t i p}} \approx-\left(\kappa \frac{d T}{d z}\right)_{T_{a r c} \leq T \leq T_{t i p}}
$$

Summing-up the two terms in eq. 6 yields:

$$
\begin{aligned}
& \left(\kappa \frac{d T}{d z}\right)_{T_{t i p}}^{2}+\frac{4}{r_{E}} \int_{\tau=T}^{\tau=T_{t i p}} q_{E} \kappa d \tau \\
& \approx\left[q_{E}\left(T_{t i p}\right)-q_{R}\right]^{2}+\frac{4}{r_{E}}\left[q_{E}\left(T_{t i p}\right)-q_{R}\right] \int_{0}^{r_{E}} q_{E}(z) d z \\
& =\left(\frac{1}{\pi r_{e}^{2}}\right)^{2}\left[\pi r_{E}^{2}\left(q_{E}\left(T_{t i p}\right)-q_{R}\right)+2 \pi r_{E} \int_{0}^{r_{E}} q_{E}(z) d z\right]^{2}-\left[\frac{2 \pi r_{E}}{\pi r_{E}^{2}} \int_{0}^{r_{E}} q_{E} d z\right]^{2}
\end{aligned}
$$

The quantity

$$
P_{i n}=\pi r_{E}^{2} q_{E}\left(T_{t i p}\right)+2 \pi r_{E} \int_{0}^{r_{E}} q_{E}(z) d z
$$


is the total power input into the electrode tip, the quantity

$$
P_{r}=q_{R}\left(T_{t i p}\right) \pi r_{E}^{2}
$$

is the radiant power, which is emitted by the end face of the electrode and

$$
P_{c}=2 \pi r_{E} \int_{0}^{r_{E}} q_{E} d z
$$

the power input into the cylinder barrel of the rod. Inserting eq. 11, eq. 12 and eq. 13 into eq. 10 yields:

$$
\left(\kappa \frac{d T}{d z}\right)_{T_{t i p}}^{2}+\frac{4}{r_{E}} \int_{\tau=T}^{\tau=T_{t i p}} q_{e} \kappa d \tau=\frac{\left(P_{i n}-P_{r}\right)^{2}-P_{c}^{2}}{\left(r_{E}^{2} \pi\right)^{2}} .
$$

The ratio:

$$
\left(\frac{P_{c}}{P_{\text {in }}-P_{r}}\right)^{2} \ll 1
$$

can be neglected very often. The integration of eq. 6 provides, neglecting $P_{c}$, a relation $z_{\text {sim }}(T)$, which can be compared with results from measurements.

$$
z_{\text {sim }}(T) \approx
$$

$$
\int_{T^{\prime}=T}^{T_{t i p}} \frac{\kappa\left(T^{\prime}\right) d T^{\prime}}{\left[\left(\frac{P_{i n}-P_{r}}{\pi r_{E}^{2}}\right)_{T_{t i p}}^{2}-\frac{4}{r_{E}} \int_{\tau=T^{\prime}}^{T_{t i p}} \epsilon_{t o t} \sigma_{S B} \tau^{4} \kappa(\tau) d \tau-2 \omega \int_{\tau=T^{\prime}}^{T_{t i p}} \rho_{M} c_{p}(\tau) \kappa(\tau)\left(\frac{\partial \tau}{\partial \varphi}\right)_{\varphi_{e}} d \tau\right]^{\frac{1}{2}}}
$$

The minus sign in eq. 6 is removed by transposing the integration limits. The integral can be executed if measured values for $(\partial T / \partial \varphi)_{\varphi_{e}}$ and estimated values for $T_{t i p}$ and $\left(P_{\text {in }}-P_{r}\right)^{2}$ are inserted.

Reliable values of $z_{\text {meas }}(T)$ between lower temperatures $T_{l}$ and upper temperatures $T_{u p}$ are provided by measurements. They can be used to determine appropriate values of $P_{i n}$ and $T_{\text {tip }}$ by searching the minimum of:

$$
f\left(P_{\text {in }}, T_{\text {tip }}\right)=\int_{T_{l}}^{T_{u p}}\left[z_{\text {meas }}(T)-z_{\text {sim }}(T)\right]^{2} d T .
$$

A consideration of the difference

$$
\left(\frac{P_{i n}-P_{r}}{r_{E}^{2} \pi}\right)^{2}-\left(\kappa \frac{d T}{d z}\right)_{T_{t i p}}^{2}-\frac{4}{r_{E}} \int_{\tau=T}^{\tau=T_{t i p}} q_{E} \kappa d \tau=\left(\frac{P_{c}}{r_{e}^{2} \pi}\right)^{2}
$$

may be necessary by an additional simulation of the electrode tip if the arc attachment on the lateral area of the electrode cannot be neglected. It becomes only zero if $P_{i n}$ is exclusively coupled into the end face of the electrode. It will be favourable to choose the experimental conditions in such a way that the difference can be neglected. 
In figure 2 two examples, one for the anodic phase and an other one for the cathodic phase, are shown for measured courses of the temperature along the electrode rod, $T(z)$, and the course of the phase variation of the temperature in dependence on $z$, $(\partial T(z) / \partial \varphi)_{\varphi_{e}}$. For comparison courses of $T\left(z_{\text {sim }}\right)$ are added which are calculated with eq. 16 and eq. 17 from the experimental data. The length of the electrode amounts to $l_{e}=20 \mathrm{~mm}$ and its diameter to $d_{E}=1 \mathrm{~mm}$, the filling gas is argon at a pressure $p=0.26 \mathrm{MPa}$. The arc is operated by a sinusoidal current with $f=10 \mathrm{~Hz}$ and an amplitude $\hat{i}=4.24 \mathrm{~A}$. In figure $2 \mathrm{a} T(z),(\partial T(z) / \partial \varphi)_{\varphi_{e}}$ and $T\left(z_{\text {sim }}\right)$ in the anodic half period for $\varphi=\pi / 4$ are presented and in figure $2 \mathrm{~b}$ in the cathodic half period for $\varphi=5 / 4 \pi$. In addition results for $T_{t i p}$ and $P_{i n}$ are given deduced from the measurements in the anodic and cathodic phase.

The relation between the variation of $P_{i n}$ and $T_{t i p}$ with $\varphi$ is controlled by the dependence of the second integral within the square root under the fraction bar in eq. 16 on $(\partial T(z) / \partial \varphi)_{\varphi_{e}}$. It takes into account the heat capacity of the electrode. A distinct variation of the temperature with $\varphi$ occurs only in the vicinity of the electrode tip, but disappears with increasing distance from the tip [22]. Its dimension is given by the penetration depth of a sinusoidal modulation of the temperature at the electrode tip $\left.\lambda_{\text {pen }}=\left(\kappa / \rho_{M} c_{p} 2 \pi f\right)^{1 / 2}\right)$. It amounts to $\lambda_{\text {pen }}=0.6 \mathrm{~mm}$ for $T_{\text {tip }}=3000 \mathrm{~K}$ and $f=10 \mathrm{~Hz}$ in qualitative agreement with the results given in figure 2 . This means that the temperature distribution $T(z)$ in the vicinity of the electrode tip has to be taken into account in eq. 17 and therefore also in all terms within the square root under the fraction bar in eq. 16 to consider the variation of $P_{i n}$ and $T_{\text {tip }}$ in dependence on $\varphi$. All terms depend very sensitively on $T(z) . P_{r}$ depends on $T_{\text {tip }}^{4}$ and the integrand of the first integral within the square root on $T^{4}$. The exaggerated values of $T_{t i p}^{4}$ in $P_{r}$ and of $T^{4}$ in the integrand may be compensated in part by too low values of $\epsilon_{\text {tot }}$. The uncertainty of $T(z)$ and $(\partial T(z, \varphi) / \partial \varphi)_{\varphi_{e}}$ in the vicinity of the electrode tip is the dominant error source of $P_{i n}(\varphi)$. Compared to this, erroneous material properties and an extension of the arc attachment onto the lateral surface of the electrode $\left(P_{c}>0\right)$ can be neglected. It is found for the examples given in figure $2 \mathrm{a}$ and $2 \mathrm{~b}$ :

$T_{\text {tip }}(\pi / 4)=(3088 \pm 30) \mathrm{K}, T_{\text {tip }}(5 / 4 \pi)=(3102 \pm 60) \mathrm{K}$.

The dependence of the courses of $P_{i n}(\varphi)$ on the erroneous values of $T(z)$, enhanced by the terms proportional to $T^{4}$ in eq. 16 , and on the erroneous values of $(\partial T(z) / \partial \varphi)_{\varphi_{e}}$ in the vicinity of the electrode tip is more pronounced than that of $T_{t i p}(\varphi)$. It is found for the examples given in figure $2 \mathrm{a} / 2 \mathrm{~b}$ :

$P_{\text {in }}(\pi / 4)=(23.1 \pm 1.3) \mathrm{W}, P_{\text {in }}(5 / 4 \pi)=(26.1 \pm 2.5) \mathrm{W}$.

The penetration depth $\lambda_{\text {pen }}$ and the amplitude of the modulation of $T(z, \varphi)$ decrease with increasing operating frequency. The penetration depth is reduced to $\lambda_{\text {pen }} \approx 60 \mu \mathrm{m}$ if the operating frequency is increased from $f_{1}=10 \mathrm{~Hz}$ to $f_{2}=1 \mathrm{kHz}$. The amplitude modulation $\Delta T_{\text {tip }}(\varphi)$, which is approximately proportional to $\Delta P_{i n} /\left(\rho_{M} c_{p} r_{E}^{2} \pi \lambda_{p e n} 2 \pi f\right)$ is reduced by the same factor $\sqrt{f_{1} / f_{2}} \cdot\left(r_{E} \pi \lambda_{\text {pen }}\right.$ represents the volume of the electrode tip in which a temperature variation takes place.) Therefore the second integral within the square root under the fraction bar in eq. 16 can be neglected and with it the 
temperature distribution in the vicinity of the electrode tip. As in [5] the course of $z_{\text {meas }}(T)$ within a region of confidence can be used to determine $T_{t i p}$ and $P_{i n}$. The results are the same as those which are obtained with the procedure given in [5]. But it has to be kept in mind that information on a different power input into the electrode within the anodic and cathodic phase may get lost if variations of the electrode temperature in dependence on phase cannot be resolved.

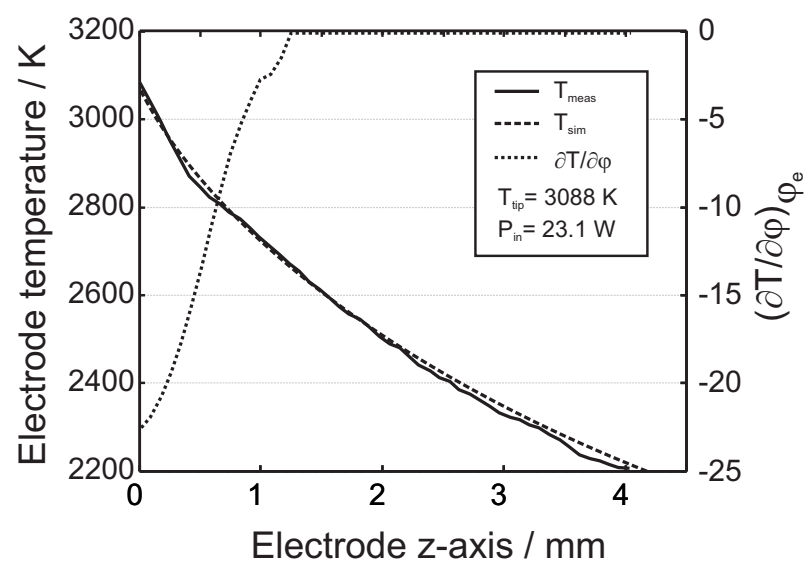

(a) anodic phase

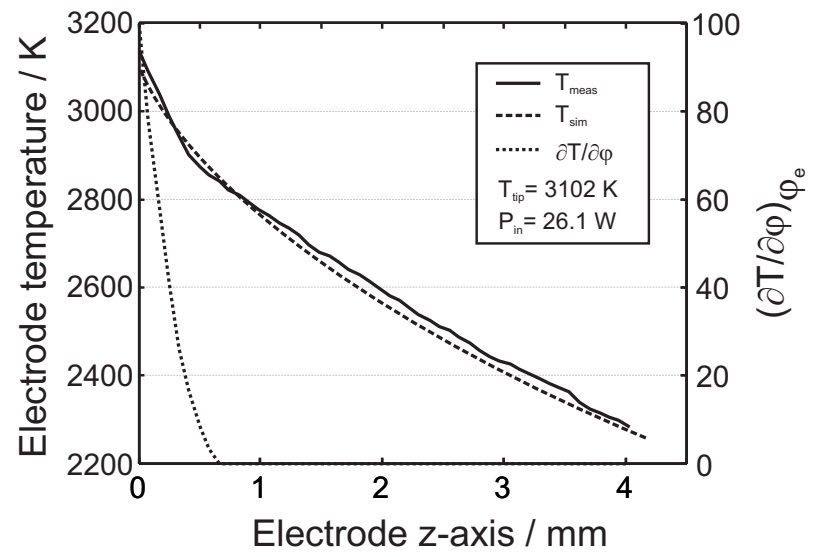

(b) cathodic phase

Figure 2. Measured and simulated temperature $T(z), T\left(z_{\text {sim }}\right)$ along the electrode z-axis at phase positions of a) $\varphi_{e}=\pi / 4$ (anodic phase) and b) $\varphi_{e}=5 / 4 \pi$ (cathodic phase) and the corresponding values $(\partial T / \partial \varphi)_{\varphi_{e}}$ for a sinusoidal current $i_{r m s}=3 \mathrm{~A}$, $f=10 \mathrm{~Hz}$. Electrode parameters: diameter $d_{E}=1 \mathrm{~mm}$, electrode length $l_{E}=20 \mathrm{~mm}$, gas: argon, pressure $p=0.26 \mathrm{MPa}$. In addition results for $T_{t i p}$ and $P_{i n}$ are given

\section{Measuring results}

Results of the electrode tip temperature $T_{\text {tip }}$ and of the power $P_{i n}$ coupled from the plasma into the electrode are presented in dependence on the phase angle $\varphi$. The pyrometric measurements are supplemented in part by $\operatorname{ESV}(\varphi)$ measurements. To investigate the influence of the current waveform on the electrode behaviour, the 
electrode is either operated with a switched dc or a sinusoidal current.

The dimensions of the electrode are $l_{E}=20 \mathrm{~mm}, d_{E}=1 \mathrm{~mm}$. The amount of the rms-current $i$ is varied between $i=3 \mathrm{~A}$ and $i=6 \mathrm{~A}$. By the special choice of parameters the formation of a spot attachment within the cathodic phase is avoided. But minor variations of the arc attachment on the electrode, also for constant macroscopic parameters, have to be considered, especially if the results of different measurements are compared. Measurements are performed at operating frequencies of $f=10,25,100,500 \mathrm{~Hz}, 1,2,5$ and $10 \mathrm{kHz}$. For frequencies up to $100 \mathrm{~Hz}$ temperature measurements are performed at 20 equidistant phase angles $\varphi_{\nu}=0.1 \pi \nu,(\nu=1-20)$ within one period. The number of measuring points is reduced in the case of $f=500 \mathrm{~Hz}$, $1 \mathrm{kHz}$ to 10 points, for $f=2 \mathrm{kHz}$ to 4 points and finally to 2 points for $f=5,10 \mathrm{kHz}$. The reduction may be justified since the amplitude modulation decreases with increasing operating frequency. In the subsequent figures the results for some frequencies are omitted to achieve a clearer representation. A documentation of all measurements can be found in the $\mathrm{PhD}$ thesis of Reinelt [33]. In the subsequent figures the measurements within the anodic half period are presented between $\varphi=0$ and $\varphi=\pi$, and those within the cathodic half period between $\varphi=\pi$ and $\varphi=2 \pi$.

\subsection{Operation by a switched dc current}

Figure 3 shows courses of $T_{\text {tip }}(\varphi)$ for different frequencies between $10 \mathrm{~Hz}$ and $10 \mathrm{kHz}$, and different amplitudes of the switched dc current. In figure 3 a courses of $T_{\text {tip }}(\varphi)$ for $i=3 \mathrm{~A}$, $f=10,25,500 \mathrm{~Hz}$ and $10 \mathrm{kHz}$ are given. For $f=10 \mathrm{~Hz}$ an approximately exponential decrease of the electrode temperature during the anodic phase by $\Delta T \geq 120 \mathrm{~K}$ is observed. The lowering is reduced a bit more than half $(\approx 65 \mathrm{~K})$ if the operating frequency is increased to $25 \mathrm{~Hz}$. The variation of $T_{\text {tip }}$ in dependence on $\varphi$ disappears to a large extent at higher frequencies, but a weak temperature increase is observed if the frequency is raised. For $f=10 \mathrm{kHz}$ the average value of the electrode tip temperature approaches $T_{t i p}=3140 \mathrm{~K}$. It may be compared with $T_{t i p} \approx 3000 \mathrm{~K}$ of the cathode [11] and $T_{t i p} \approx 2780 \mathrm{~K}$ of the anode [16], if an electrode with the same parameters is operated with a dc current of $I=3 \mathrm{~A}$. The courses of $T_{\text {tip }}(\varphi)$ for $i=4 \mathrm{~A}, f=10,25,500 \mathrm{~Hz}$ and $10 \mathrm{kHz}$ in figure $3 \mathrm{~b}$ are qualitatively the same. The temperature level is raised approximately by $50 \mathrm{~K}$, but the temperature modulation is almost halved. For $f=10 \mathrm{kHz}$ the average value of the electrode tip temperature is $T_{t i p} \approx 3185 \mathrm{~K}$ compared to $T_{t i p} \approx 3060 \mathrm{~K}$ of the cathode [11] and $T_{t i p} \approx 3100 \mathrm{~K}$ of the anode if the electrode is operated with a dc current of the same amplitude. For $i=6 \mathrm{~A}, f=10,25 \mathrm{~Hz}$ an approximately exponentially declining increase of $T_{\text {tip }}(\varphi)$ is observed within the anodic phase and an approximately exponential decrease within the cathodic phase at low operation frequencies as is shown in figure $3 \mathrm{c}$. The temperature rises at the end of the anodic phase to $T_{\text {tip }} \approx 3330 \mathrm{~K}$ for $f=10 \mathrm{~Hz}$. It has to be compared with $T_{\text {tip }} \approx 3700 \mathrm{~K}$ which is realized if the electrode is operated as an anode with a dc current [16] (fig. 1). In this case the electrode tip would be melted. At the end of the cathodic phase the temperature is reduced 
to $T_{\text {tip }} \approx 3275 \mathrm{~K}$. For $f \geq 100 \mathrm{~Hz}$ the temperature modulation disappears to a large extent and a nearly constant electrode tip temperature $T_{t i p} \approx 3280 \mathrm{~K}$ is reached. It is different to $i=3 \mathrm{~A}$ and $i=4 \mathrm{~A}$ less than the average between the cathode temperature $T_{\text {tip }} \approx 3140 \mathrm{~K}\left[11\right.$ ](fig. 8 ) and the anode temperature $T_{\text {tip }} \approx 3700 \mathrm{~K}$ if the electrode is operated with a dc current of the same amplitude.

It was shown previously by experiments with de currents that at low currents the temperature of the cathode tip is higher than that of the anode tip, but that at higher currents the anode becomes hotter than the cathode. In case of the present experimental conditions both temperatures become equal, $T_{t i p} \approx 3040 \mathrm{~K}$, for a dc current of $I=3.6 \mathrm{~A}$ [16] (fig. 19), [19] (fig. 16). In case of an operation with a $f=10 \mathrm{~Hz}$ switched dc current the point at which the anode temperature passes the cathode temperature is displaced to a current amplitude of $i=4.8 \mathrm{~A}$ and $T_{\text {tip }}=3240 \mathrm{~K}$. The displacement to a higher current reflects the increase of the cathode temperature and the increase of the anode temperature at low currents and its lowering at higher currents, so that the course of the anode temperature $T_{\text {tip }}(i)$ becomes flatter, with increasing operating frequency.

Figure 4 gives the courses of the total power input $P_{i n}(\varphi)$ into the electrode for the same discharge parameters as in figure 3. The courses of $P_{i n}(\varphi)$ for $i=3 \mathrm{~A}$ and $i=4 \mathrm{~A}$ in figure $4 \mathrm{a}$ and figure $4 \mathrm{~b}$ show the same tendencies as those of $T_{t i p}(\varphi)$, a decrease within the anodic phase and a steep increase at the beginning of the cathodic phase. The average power input for $f=10 \mathrm{kHz}, i=3 \mathrm{~A}$ and $i=4 \mathrm{~A}$, amounts to $P_{\text {in }}=27 \mathrm{~W}$ and $P_{i n}=28.5 \mathrm{~W}$, compared to the power input into an electrode operated with a dc current of the same amplitude, $P_{i n}=27 \mathrm{~W}$ and $P_{i n}=30 \mathrm{~W}$ into the cathode, [7] (fig. 12), [11] (fig. 13), $P_{\text {in }} \approx 20 \mathrm{~W}$ and $P_{\text {in }} \approx 25 \mathrm{~W}$ into the anode [16] (fig. 1). For $i=6 \mathrm{~A}$, a modulation in dependence on $\varphi$ is still visible, but quite weak. The reason is that the power input into the cathodic and anodic half period is only marginally different. The average power input for $f=10 \mathrm{kHz}$ amounts to $P_{\text {in }} \approx 30.5 \mathrm{~W}$. If an electrode with the same parameters is operated with a dc current of $i=6$ A the power input into the cathode is $P_{\text {in }}=31 \mathrm{~W}$ [7] (fig. 12) and into the anode $P_{\text {in }}=37.5 \mathrm{~W}$ [16].

In figure 5 the courses of the tip temperature $T_{\text {tip }}(\varphi)$ and of the power input $P_{\text {in }}(\varphi)$ measured with switched dc currents of $i=3,4$ and $6 \mathrm{~A}, f=10 \mathrm{~Hz}$ and $f=10 \mathrm{kHz}$ are compared. The increase of $T_{t i p}$ and $P_{i n}$ with increasing current amplitude meets the expectation. A variation of $T_{t i p}$ by less than $5 \%$ and of $P_{i n}$ by less than $16 \%$ in dependence on $\varphi$ are found for $f=10 \mathrm{~Hz}$. A detailed description of the modulation was already given. For $f=10 \mathrm{kHz}$ the dependence of $T_{\text {tip }}$ on $\varphi$ can be neglected. But it is not sure that the difference between the power inputs into the cathodic and anodic phase really disappears. In the cathodic phase a bit higher values of $T_{t i p}$ are found than in case of dc operation.

Supplementary in figure 6 courses of the $\operatorname{ESV}(\varphi)$ for a switched dc current of $i=3 \mathrm{~A}$ and operating frequencies of $f=10,500 \mathrm{~Hz}, 5$ and $20 \mathrm{kHz}$ are given. They clearly indicate for frequencies up to $500 \mathrm{~Hz}$ a commutation peak after current zero crossing which can be attributed to the cathode fall $U_{c}(\varphi)$. It represents an enhanced heating of the cathode for a short time after current zero crossing by which a reduced power input 


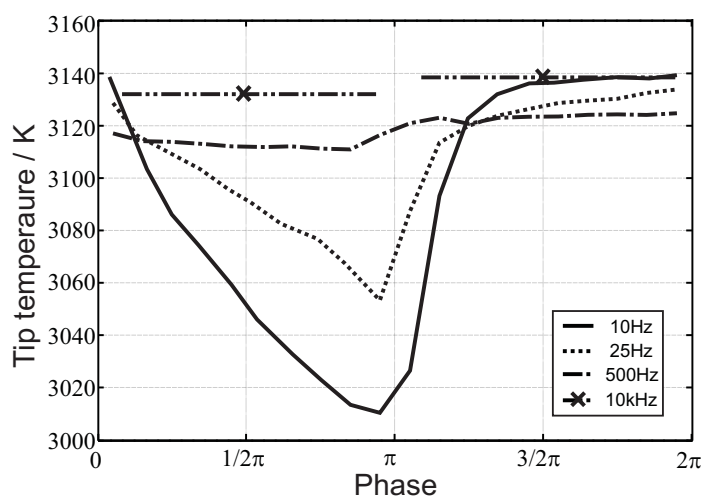

(a) $\mathrm{i}=3 \mathrm{~A}$

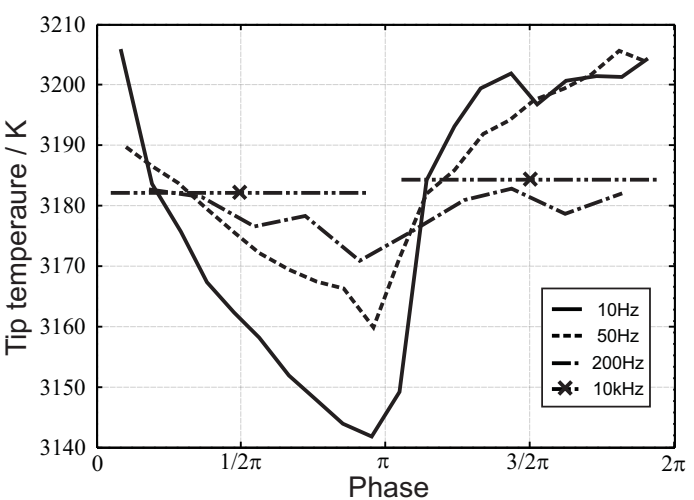

(b) $\mathrm{i}=4 \mathrm{~A}$

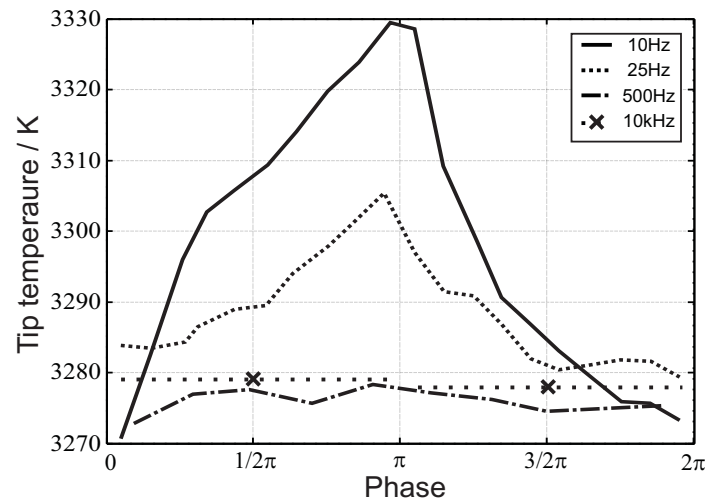

(c) $\mathrm{i}=6 \mathrm{~A}$

Figure 3. Spectroscopically measured electrode tip temperature for various frequencies. The first half period represents the anodic phase and the second half period the cathodic phase. Parameters: electrode diameter $d_{E}=1 \mathrm{~mm}$, electrode length $l_{E}=20 \mathrm{~mm}$, switched dc with $i=3,4$ and $6 \mathrm{~A}$, gas: argon, pressure $p=0.26 \mathrm{MPa}$

within the anodic phase is compensated. It is shown in figure $4 \mathrm{a}$ up to $f=500 \mathrm{~Hz}$. At higher operating frequencies the difference between the energy input within the anodic and cathodic phase may be too small to be compensated by a detectable commutation peak. The course of the $E S V$ for $f=20 \mathrm{kHz}$ shows that a switched dc current is no longer realized by the amplifier with satisfying accuracy at this frequency. A peak of $P_{i n}(\varphi)$ corresponding to the commutation peak could not be resolved by the pyrometric measurements in figure $4 \mathrm{a}$. The amplitude of the commutation peaks diminishes if the current is raised to $i=4 \mathrm{~A}$ indicating that the difference between the energy input within the anodic and cathodic phase is reduced. The commutation peaks after current zero crossing have cleared away for $i=6 \mathrm{~A}$.

\subsection{Operation by a sinusoidal current}

Figure 7 presents the courses of $T_{\text {tip }}(\varphi)$ for different frequencies between $10 \mathrm{~Hz}$ and $10 \mathrm{kHz}$ and different rms-values of the sinusoidal current. In figure 7a courses of $T_{\text {tip }}(\varphi)$ for $i_{r m s}=3 \mathrm{~A}, f=10,25,500 \mathrm{~Hz}$ and $10 \mathrm{kHz}$ are given. For $f=10 \mathrm{~Hz}$ a quite mono- 


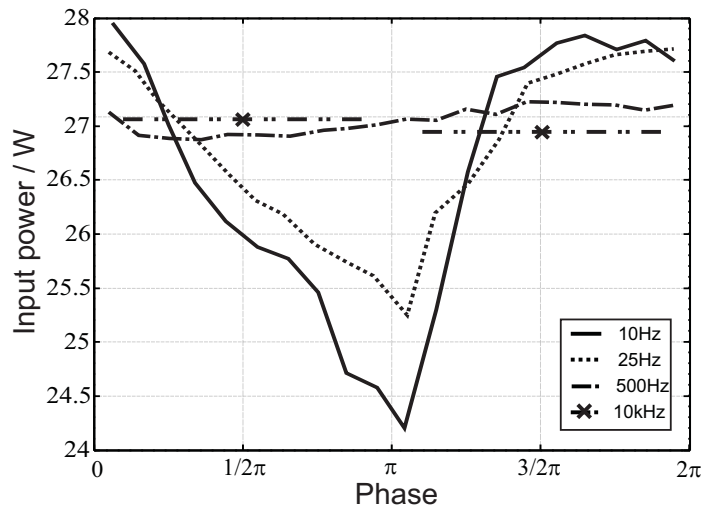

(a) $\mathrm{i}=3 \mathrm{~A}$

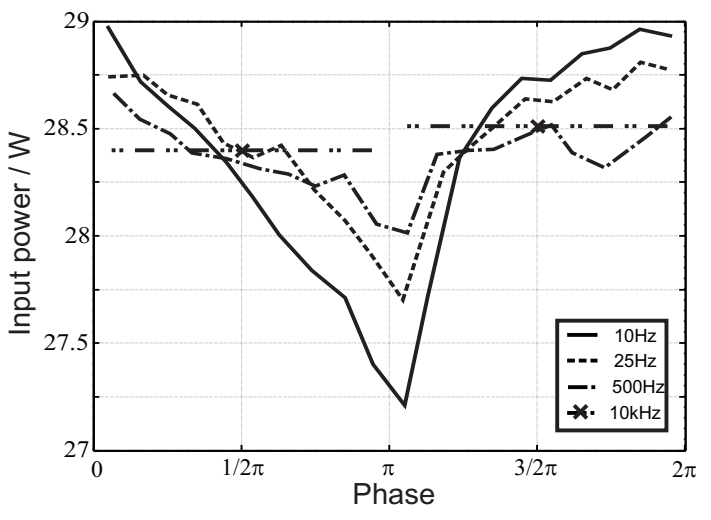

(b) $\mathrm{i}=4 \mathrm{~A}$

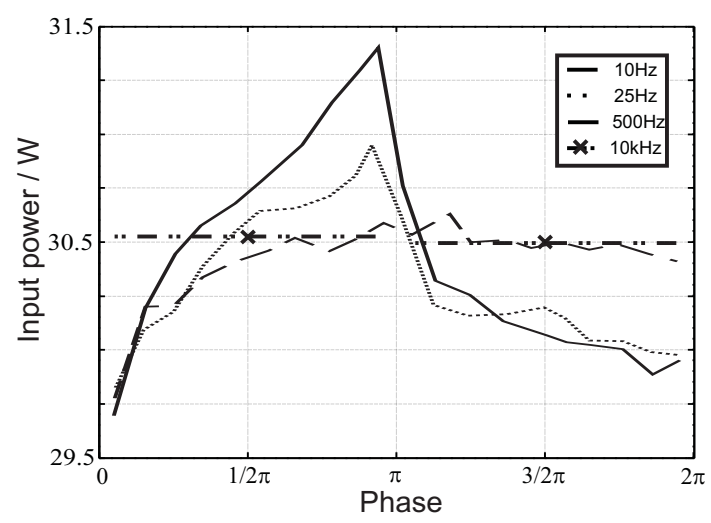

(c) $\mathrm{i}=6 \mathrm{~A}$

Figure 4. Electrode input power for various frequencies. The first half period represents the anodic phase and the second half period the cathodic phase. Parameters: electrode diameter $d_{E}=1 \mathrm{~mm}$, electrode length $l_{E}=20 \mathrm{~mm}$, switched dc with $i=3$, 4 and $6 \mathrm{~A}$, gas: argon, pressure $p=0.26 \mathrm{MPa}$

tonic decrease of the electrode temperature during the anodic phase to a minimum temperature $T_{\text {tip }} \approx 3050 \mathrm{~K}$ at $\varphi=\pi$ is observed. The decrease is disturbed by a weak maximum at $\varphi>\pi / 2$. During the cathodic phase a nearly sinusoidal increase of the tip temperature takes place between $\varphi=\pi$ and $\varphi \approx 1.67 \pi$ to $T_{\text {tip }} \approx 3200 \mathrm{~K}$ and a subsequent approximately sine-square-decrease by $\Delta T \approx 70 \mathrm{~K}$ between $\varphi \approx 1.67 \pi$ and $\varphi=2 \pi$. The amplitude of the modulation is decreased with increasing frequency. It is to a large extent removed for $f=10 \mathrm{kHz}$, but simultaneously the average value of $T_{\text {tip }}$ is raised by several ten Kelvin. It comes up to $T_{t i p} \approx 3200 \mathrm{~K}$ for $f=10 \mathrm{kHz}$ which is only a bit higher than the corresponding value $T_{t i p} \approx 3140 \mathrm{~K}$ in the case of an operation with a switched dc current but distinctly higher than $T_{t i p} \approx 3000 \mathrm{~K}$ in case of an operation with a de current of $I=3 \mathrm{~A}$.

The courses of $T_{t i p}(\varphi)$ for $i_{r m s}=4 \mathrm{~A}$ in figure $7 \mathrm{~b}$ show for lower operating frequencies different to those for $i_{r m s}=3 \mathrm{~A}$ a well developed second temperature maximum within the anodic half period. It is distinctly lower than the maximum 


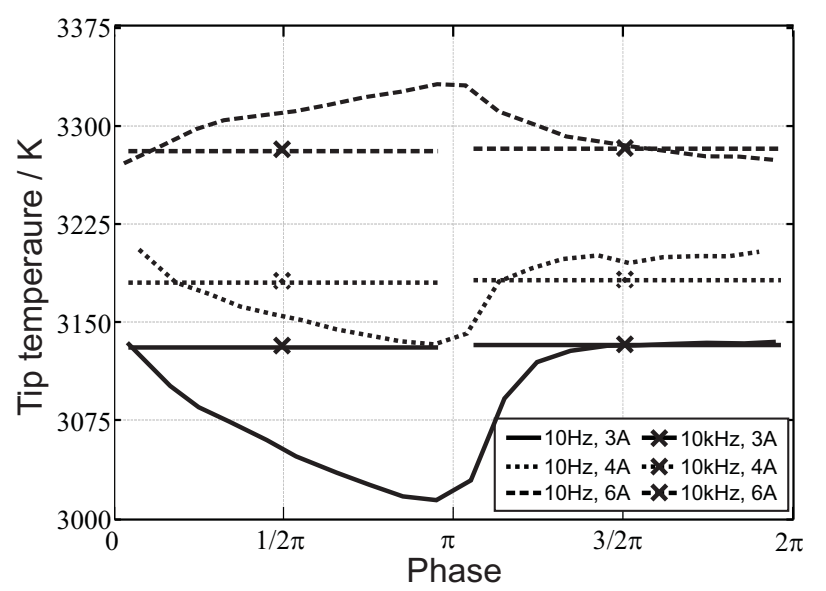

(a) Tip temperature

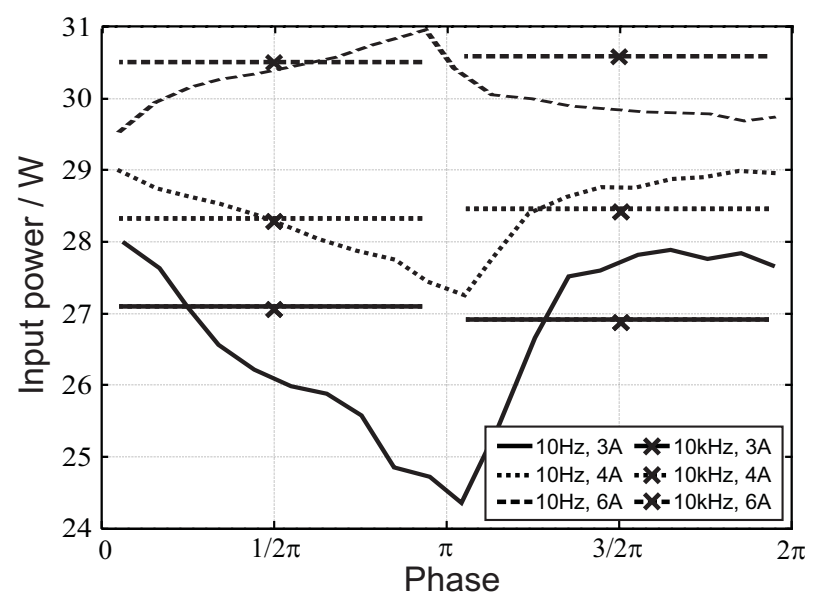

(b) Input power

Figure 5. Comparison of the electrode tip temperature and input power for a low and a high frequency for currents of $i=3,4$ and $6 \mathrm{~A}$. The first half period represents the anodic phase and the second half period the cathodic phase. Parameters: electrode diameter $d_{E}=1 \mathrm{~mm}$, electrode length $l_{E}=20 \mathrm{~mm}$, switched dc, gas: argon, pressure $p=0.26 \mathrm{MPa}$

within the cathodic half period. The difference between the two maxima amounts to $\Delta T \approx 80 \mathrm{~K}$ for $10 \mathrm{~Hz}$. It is delayed with respect to the anodic current maximum as well as the subsequent minimum with respect to current zero crossing and the cathodic temperature maximum with respect to the cathodic current maximum. The temperature level is raised approximately by $40 \mathrm{~K}$ compared to $i_{r m s}=3 \mathrm{~A}$. The variation of $T_{t i p}$ in dependence on $\varphi$ fades away at higher frequencies. It is accompanied by a weak increase of the average tip temperature. It rises to $T_{t i p} \approx 3240 \mathrm{~K}$ compared to $T_{t i p} \approx 3185 \mathrm{~K}$ for an operation with a switched dc current and a cathode temperature $T_{\text {tip }}=3060 \mathrm{~K}$ for an operation with the corresponding dc current.

The courses of $T_{t i p}(\varphi)$ for $i_{r m s}=6 \mathrm{~A}$ in figure $7 \mathrm{c}$ are characterized by a temperature increase within the anodic phase with respect to the cathodic phase. The temperature difference between the two maxima has reduced to $\Delta T \approx 20 \mathrm{~K}$ for $10 \mathrm{~Hz}$. In the case 


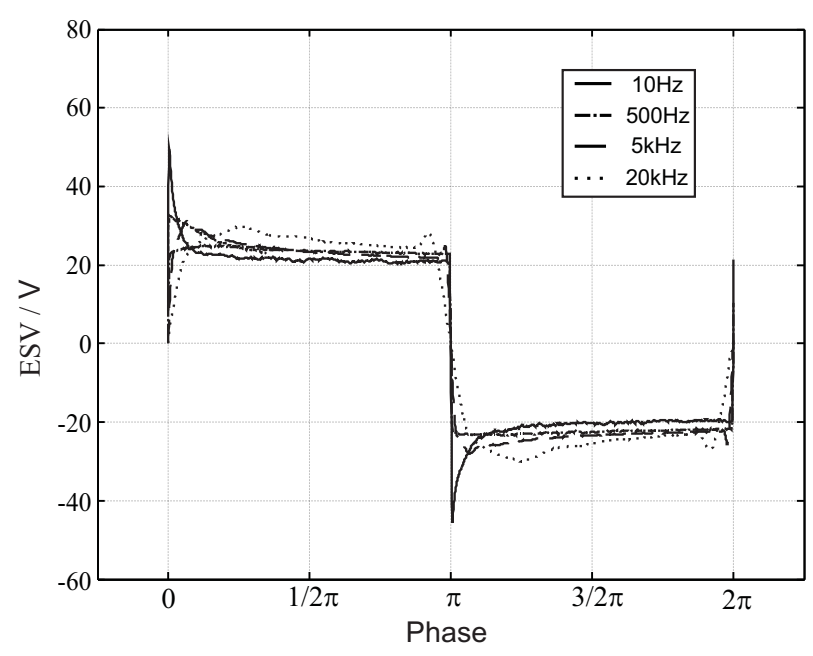

(a) switched-DC

Figure 6. $\operatorname{ESV}(\varphi)$ measurements for a switched dc current of $i=3 \mathrm{~A}, f=10,500 \mathrm{~Hz}$, 5 and $20 \mathrm{kHz}$. Electrode parameters: diameter $d_{E}=1 \mathrm{~mm}$, electrode length $l_{E}=$ $20 \mathrm{~mm}$, gas: argon, pressure $p=0.26 \mathrm{MPa}$

of an operation with a switched dc current with an amplitude $i=6$ A the temperature is higher within the anodic phase than within the cathodic phase. The average tip temperature for $f=10 \mathrm{kHz}$ has risen to $T_{\text {tip }} \approx 3410 \mathrm{~K}$ compared to $T_{\text {tip }} \approx 3280 \mathrm{~K}$ for an operation with a switched dc current. It is lower than the tip temperature of the anode $T_{\text {tip }}=3700 \mathrm{~K}$ and higher than the tip temperature of the cathode $T_{t i p}=3140 \mathrm{~K}$ being operated with a dc current $I=6 \mathrm{~A}$.

In figure 8 courses of the total power input $P_{i n}(\varphi)$ into the electrode for the same discharge parameters as in figure 7 are given. The courses of $P_{i n}(\varphi)$ for $i_{r m s}=3 \mathrm{~A}$ is quite similar to that of $T_{t i p}(\varphi)$, but the modulation of $P_{i n}(\varphi)$ is more pronounced than that of $T_{\text {tip }}(\varphi)$. The same is true for the courses of $P_{i n}(\varphi)$ given for $i_{r m s}=4 \mathrm{~A}$ and $i_{r m s}=6 \mathrm{~A}$. The average power input for $i_{r m s}=3 \mathrm{~A}, f=10 \mathrm{kHz}$ is $P_{\text {in }} \approx 29.5 \mathrm{~W}$. It may be compared with the average power input of $P_{i n}(\varphi) \approx 27 \mathrm{~W}$ in the case of an operation with a switched dc current of $i=3 \mathrm{~A}$ and compared with the power input $P_{\text {in }} \approx 27 \mathrm{~W}$ into the cathode and $P_{\text {in }} \approx 20 \mathrm{~W}$ into the anode being operated with a dc current $I=3 \mathrm{~A}$. The average power input for $i_{r m s}=4 \mathrm{~A}, i_{r m s}=6 \mathrm{~A}, f=10 \mathrm{kHz}$ is $P_{\text {in }} \approx 32.5 \mathrm{~W}$ and $P_{\text {in }} \approx 34 \mathrm{~W}$ compared to $P_{\text {in }} \approx 28.5 \mathrm{~W}$ and $P_{i n} \approx 30.5 \mathrm{~W}$ in case of an operation with the corresponding switched dc currents and the power input into the cathode $P_{\text {in }}=30 \mathrm{~W}, P_{i n}=31 \mathrm{~W}$ for the corresponding dc currents.

In figure 9 courses of the tip temperature $T_{t i p}(\varphi)$ and of the power input $P_{i n}(\varphi)$ are compared for different sinusoidal currents of $i_{r m s}=3,4$ and $6 \mathrm{~A}, f=10 \mathrm{~Hz}$ and $f=10 \mathrm{kHz}$. The increase of $T_{\text {tip }}$ and $P_{\text {in }}$ with current is in agreement with measurements performed with dc and switched dc currents. $T_{\text {tip }}$ varies for $f=10 \mathrm{~Hz}$ in dependence on $\varphi$ by less than $6 \%$ and $P_{\text {in }}$ up to $50 \%$. A detailed discussion of the modulation was already given in preceding paragraphs. For $f=10 \mathrm{kHz}$ the variation of $T_{\text {tip }}$ on $\varphi$ has 
faded away. In the cathodic phase the values of $T_{t i p}$ and $P_{i n}$ are elevated with respect to measurements at electrodes being operated with a dc current.

As a supplement in figure 10 courses of $\operatorname{ESV}(\varphi)$ for a sinusoidal current of $i_{r m s}=3 \mathrm{~A}$ and operating frequencies of $f=10,500 \mathrm{~Hz}, 5$ and $20 \mathrm{kHz}$ are given. They clearly demonstrate for frequencies up to $500 \mathrm{~Hz}$ an uplifting of the $\operatorname{ESV}(\varphi)$ after current zero crossing compared to the sinusoidal waveform of $i(\varphi)$ and a corresponding reduction in front of the current maximum. It can be attributed to the cathode fall $U_{c}(\varphi)$ indicating an enhanced heating of the cathode after current zero crossing by which a reduced power input within the anodic phase is compensated. Correspondingly a lower heating in the vicinity of the current maximum is observed. The $E S V(\varphi)$ approaches to a large extent the sinusoidal current waveform for $f>500 \mathrm{~Hz}$.

\subsection{Summary of measuring results}

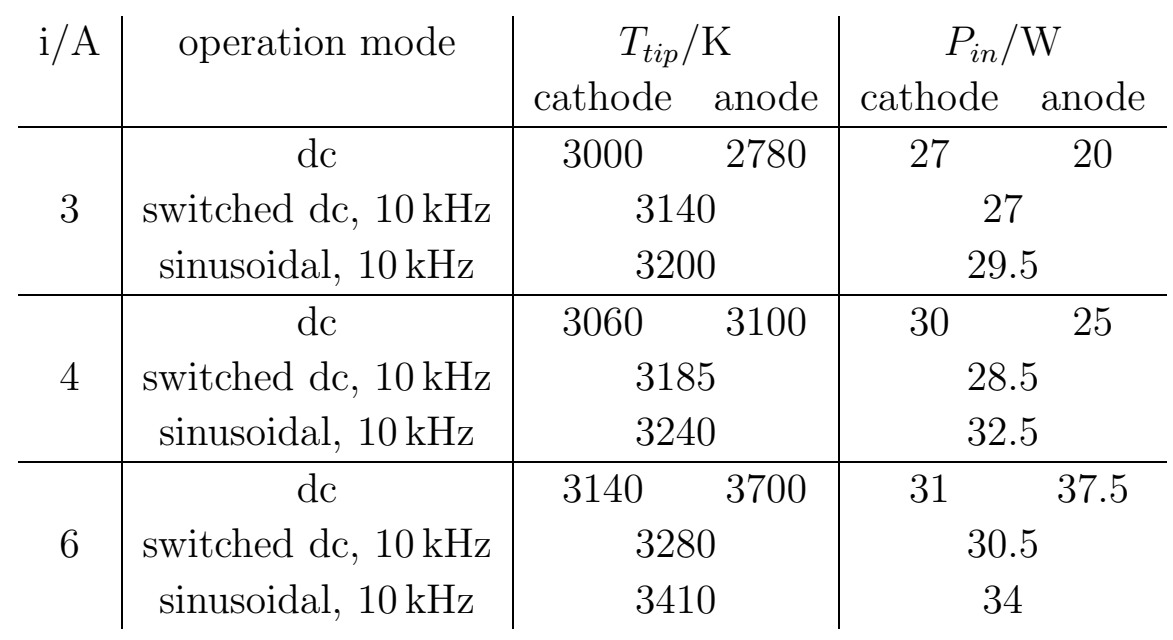

Table 1. $T_{t i p}$ and $P_{i n}$, being measured with a dc, $10 \mathrm{kHz}$ switched dc and a $10 \mathrm{kHz}$ sinusoidal current of $i_{r m s}=3,4$ and $6 \mathrm{~A}$ at the cathode and anode.

In table 1 a survey of $T_{\text {tip }}$ and $P_{\text {in }}$ are given, being measured with dc currents, $10 \mathrm{kHz}$ switched dc and $10 \mathrm{kHz}$ sinusoidal currents of $i_{r m s}=3,4$ and $6 \mathrm{~A}$.

The results clearly demonstrate for a power supply of the electrode with high operating frequencies a connection to the dc operation of the cathode. It is closer in case of an operation with a switched dc current than in case of an operation with a sinusoidal current.

\subsection{Discussion of the measuring errors}

The real values of $T_{t i p}(\varphi)$ for a special choice of current and frequency may deviate from the values given in figure 3 and figure 7 by $\leq 100 \mathrm{~K}$ due to the uncertainty of $\varepsilon(\lambda, T)$ at the electrode tip. It should be mentioned that the value of $T_{t i p}$ given in figure 3 and figure 7 are not the maximum temperatures $T_{\max }$ obtained by measurements but by an 


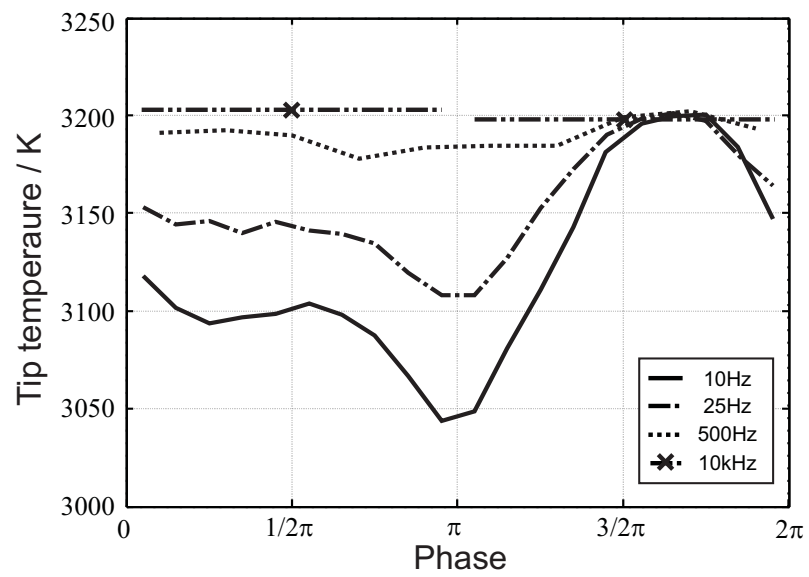

(a) $\mathrm{i}=3 \mathrm{~A}$

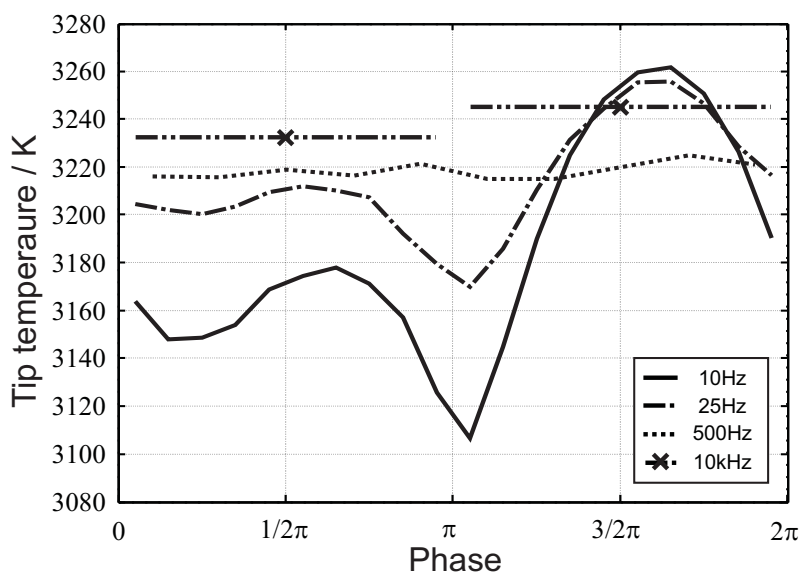

(b) $\mathrm{i}=4 \mathrm{~A}$

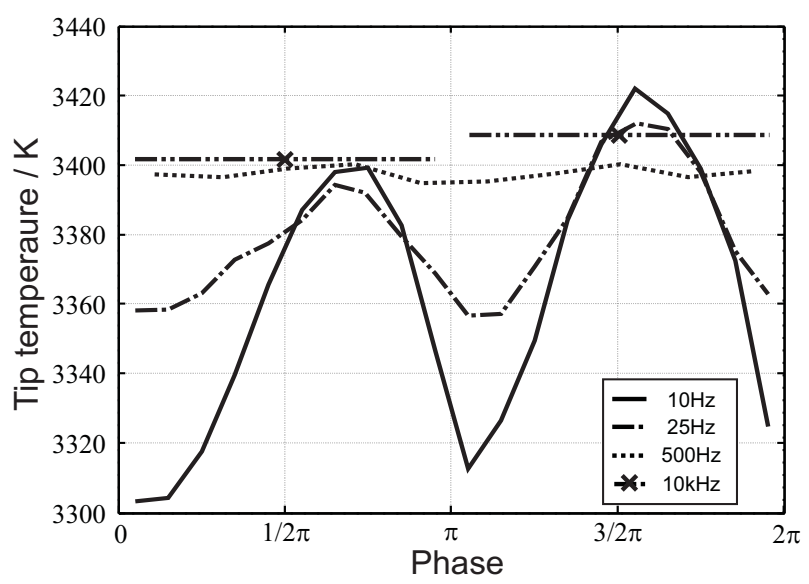

(c) $\mathrm{i}=6 \mathrm{~A}$

Figure 7. Spectroscopically measured electrode tip temperature for various frequencies. The first half period represents the anodic phase and the second half period the cathodic phase. Parameters: electrode diameter $d_{E}=1 \mathrm{~mm}$, electrode length $l_{E}=20 \mathrm{~mm}$, sin-wave with $i_{r m s}=3,4$ and $6 \mathrm{~A}$, gas: argon, pressure $p=0.26 \mathrm{MPa}$ 


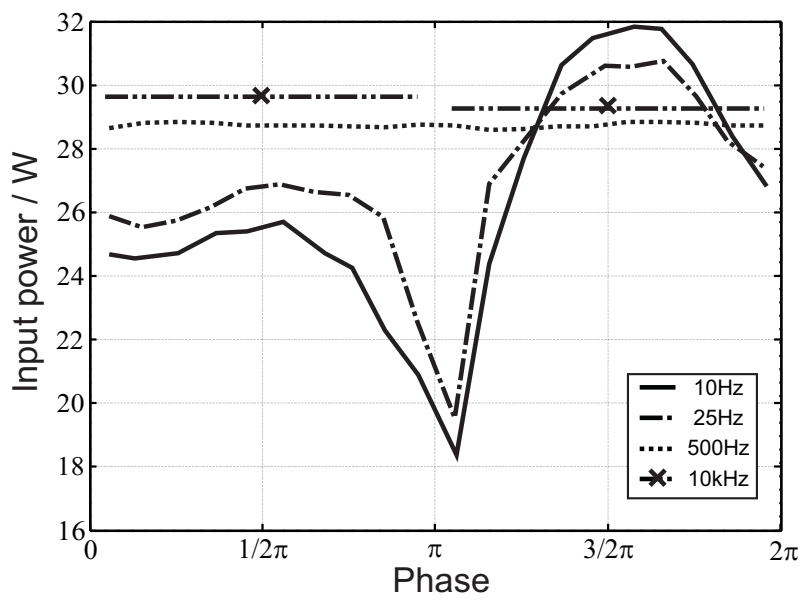

(a) $\mathrm{i}=3 \mathrm{~A}$

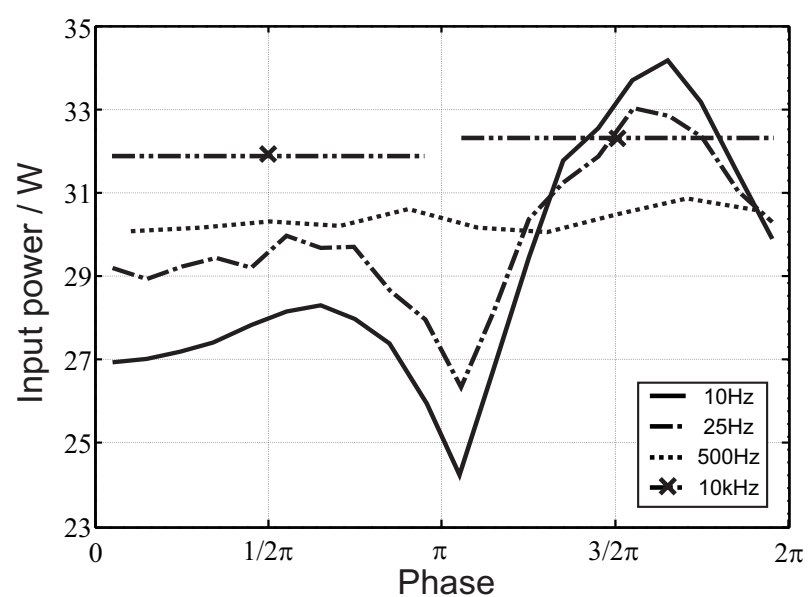

(b) $\mathrm{i}=4 \mathrm{~A}$

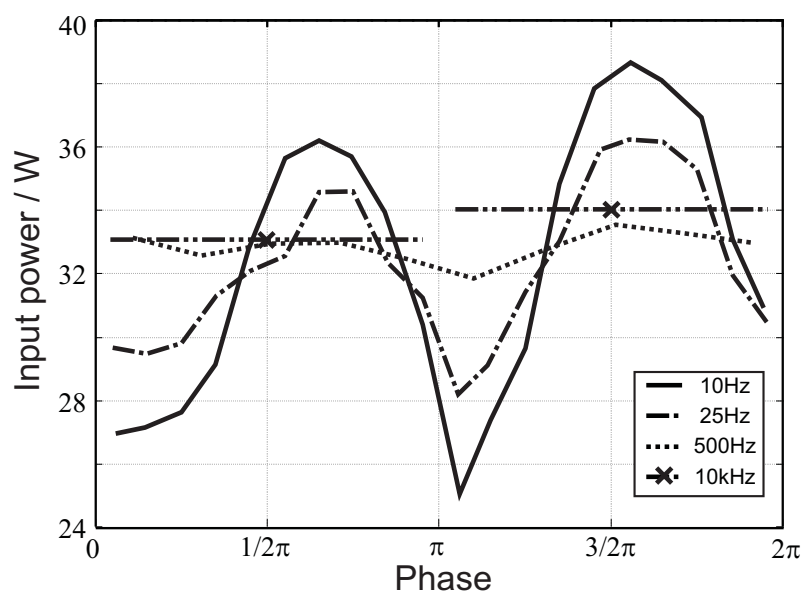

(c) $\mathrm{i}=6 \mathrm{~A}$

Figure 8. Electrode input power for various frequencies. The first half period represents the anodic phase and the second half period the cathodic phase. Parameters: electrode diameter $d_{E}=1 \mathrm{~mm}$, electrode length $l_{E}=20 \mathrm{~mm}$, sin-wave with $i_{r m s}=3$, 4 and $6 \mathrm{~A}$, gas: argon, pressure $p=0.26 \mathrm{MPa}$ 


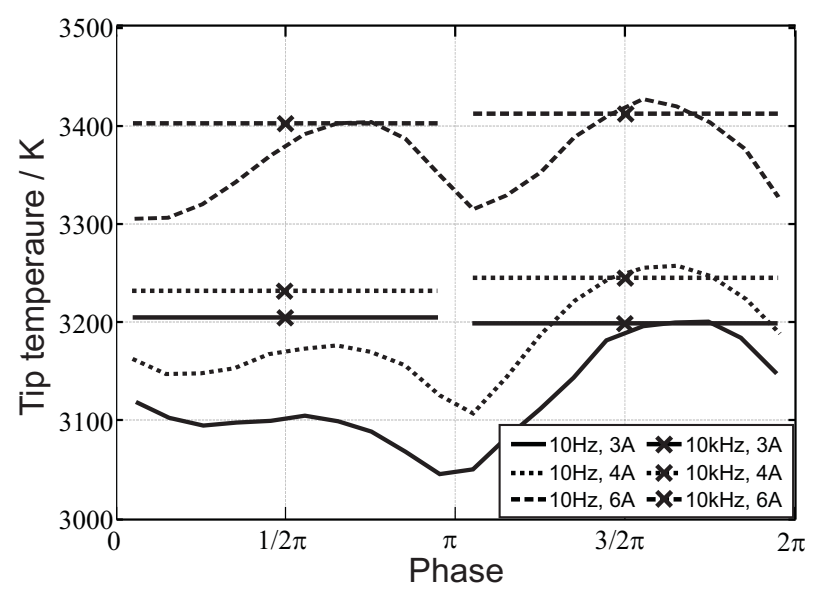

(a) Tip temperature

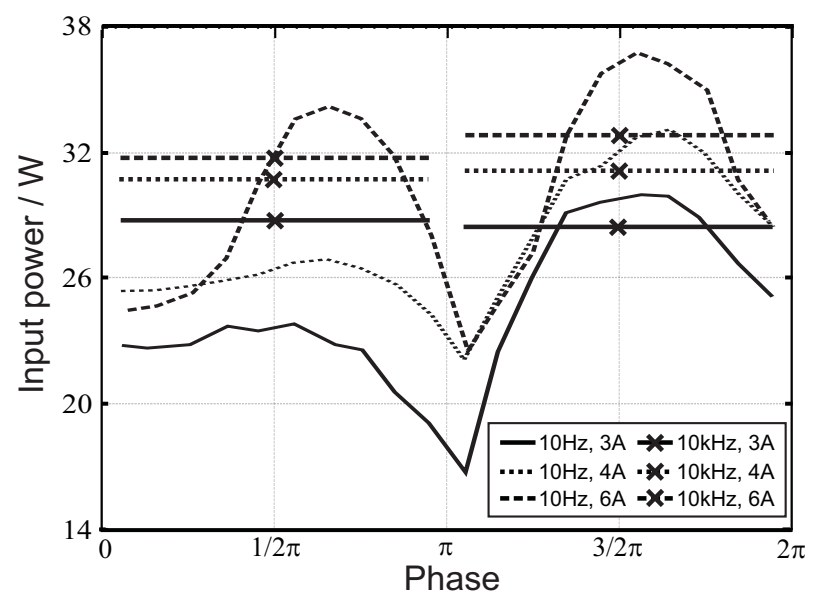

(b) Input power

Figure 9. Comparison of the electrode tip temperature and input power for a low and a high frequency for currents of $i=3,4$ and $6 \mathrm{~A}$. The first half period represents the anodic phase and the second half period the cathodic phase. Parameters: electrode diameter $d_{E}=1 \mathrm{~mm}$, electrode length $l_{E}=20 \mathrm{~mm}$, sin-wave, gas: argon, pressure $p=0.26 \mathrm{MPa}$

analysis of the measuring data $T(z)$ with eq. 16 and eq. 17 which delivers $T_{\text {tip }} \leq T_{\max }$. Two examples are given in figure 2 . The relative courses of $T_{t i p}(\varphi)$ are definitely more precise than the absolute values. A raw indication of the relative measuring errors is given by the scatter of the measuring points in figure 3 and figure 7 . They are in the order of $10 \mathrm{~K}$.

The variation of $T_{t i p}$ in dependence on $\varphi$ for different currents and frequencies corresponds to expectation. E. g. the courses of $T_{\text {tip }}(\varphi)$ in figure 3 and figure 7 clearly show a lag in phase with respect to $i(\varphi)$. The courses of $T_{t i p}(\varphi)$ will be interpreted in detail subsequently. But the courses of $P_{i n}(\varphi)$ presented in figure 4 and figure 8 are doubtful. $P_{i n}(\varphi)$ should lead in phase with respect to $T_{\text {tip }}(\varphi)$ at least for $f=10 \mathrm{~Hz}$ and $25 \mathrm{~Hz}$. But the results given in figure 4 and figure 8 do not show the expected phase shift. E. g. the courses of $T_{t i p}(\varphi)$ in figure 3 produced by a switched dc current indicate a 


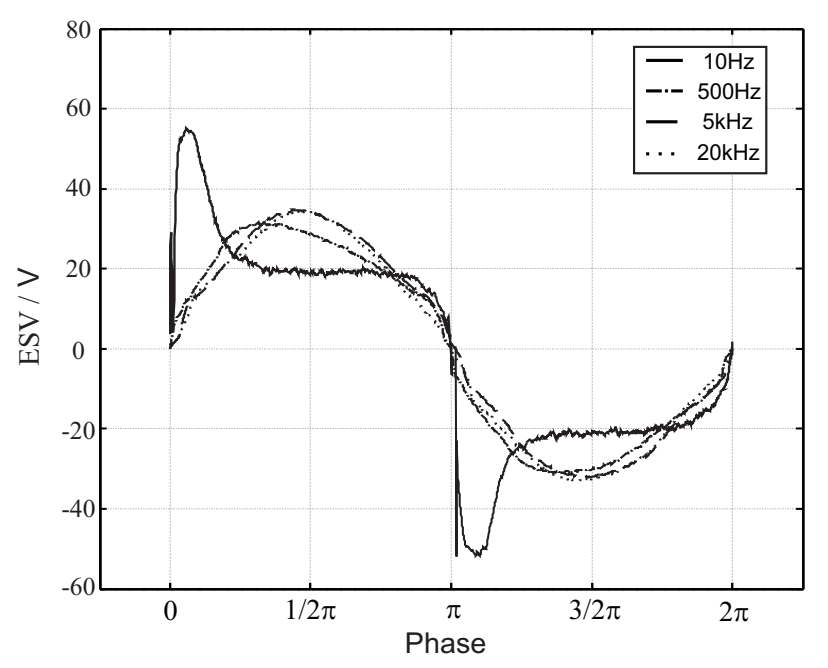

(a) $\sin$

Figure 10. $\operatorname{ESV}(\varphi)$ measurements for a sinusoidal current $i_{r m s}=3 \mathrm{~A}, f=$ $10,500 \mathrm{~Hz}, 5$ and $20 \mathrm{kHz}$. Electrode parameters: diameter $d_{E}=1 \mathrm{~mm}$, electrode length $l_{E}=20 \mathrm{~mm}$, gas: argon, pressure $p=0.26 \mathrm{MPa}$

more or less rectangular modulation of the power input $P_{i n}(\varphi)$ which is not confirmed by the results given in figure 4. Moreover it is doubtful whether the differences between the power input within the cathodic and anodic phase really disappears at higher operating frequencies since the corresponding temperature measurements at the electrode tip are quite insufficient. Also the vanishing of the commutation peak after current zero crossing of the $E S V(\varphi)$ at higher operating frequencies is not a safe indication that the power input into the anodic phase has adapted to the power input into the cathodic phase. Another result being doubtful are the high values of $P_{i n}(i=0)$ in figure 8 . The reason for the doubtful courses of $P_{i n}(\varphi)$ is presumably insufficient data for $\partial T / \partial \varphi$ which have to be measured between $0<z<0.6 \mathrm{~mm}$ beneath the electrode tip. But the average values of $P_{i n}$ which were obtained at higher operating frequencies are quite reliable. In spite of the general reservation some information given in figure 4 and figure 8 on $P_{\text {in }}(\varphi)$ are useful, namely its dependence on $i_{r m s}$, waveform and operating frequency and the relation between these parameters and the different power input into the cathodic and anodic phase.

A higher phase resolution may only be achieved by a temperature measuring technique, which is to a large extend independent of the incorrect spectral emissivity $\epsilon$ at the electrode tip, e. g. by a two or multi wavelength pyrometry. This would be rather expensive. It will be shown subsequently that the interpretation of the results is not essentially hampered by the questionable courses of $P_{i n}(\varphi)$. 


\section{Interpretation of the measuring results}

\subsection{General consideration}

In the case of a periodic power input into the electrode the input power $P_{\text {in }}$ is balanced not only by the power emitted by thermal radiation of its surface $P_{\text {rad }}$ and the power removed by heat conduction $P_{\text {cond }}$ but also by the temporal change of the heat content of the electrode $d Q_{c} / d t$ :

$$
P_{i n}(t)=P_{r a d}(t)+P_{c o n d}(t)+\frac{d Q_{c}(t)}{d t}=P_{r a d}(\varphi)+P_{c o n d}(\varphi)+\omega \frac{d Q_{c}(\varphi)}{d \varphi}(19)
$$

The relation is already given in equation 4 by a partial differential equation in dependence on $\varphi$ and $z$.

The coupling between states of the electrode and its plasma boundary layer at different moments is mainly brought about by $d Q_{c} / d t$. It applies, especially in the case of a switched dc operation, to the coupling between the cathodic and the anodic phase. As a consequence, at low currents, the electrode temperature may be much higher and at high currents much lower within the anodic phase than the temperature of an anode operated with a dc current of the same amplitude [16, 19]. Moreover the coupling prevents, in the case of an operation with a sinusoidal current, a steep drop of the electrode temperature at current zero crossing. In general the electrode temperature remains elevated by its heat capacity at moments of low power input and reduced at moments of high power input compared to an operation with a dc current. It decouples the current density of thermionic electron emission of the electrode $j_{\text {em }}\left(T_{\text {electrode }}\right)$ from the total current density $j$ in front of the electrode surface so that a strong thermionic emission may be present even in the anodic phase or in the vicinity of current zero crossing. The surplus of thermionically emitted electrons $j_{\text {em }}$ returns to the electrode in the anodic phase with a thermal energy, which corresponds to the temperature of the plasma electrons $T_{e s}$ within the sheath in front of the electrode. This means that it is elevated compared to the thermal energy of the emitted electrons which is determined by the temperature of the electrode $T_{\text {electrode }}<T_{e s}$. It accomplishes a power transfer from the plasma boundary layer to the electrode given by

$$
p_{\text {em }}=j_{\text {em }}\left(\frac{2 k_{B} T_{e s}}{e}-\frac{2 k_{B} T_{\text {electrode }}}{e}\right)
$$

as was already explained in detail in [19]. It withdraws energy from the plasma boundary layer in front of the electrode and transfers it to the anode. It may be supplied not only by an enhanced electrical power input into the plasma sheath but also by a reduction of the energy content of the plasma boundary layer per unit area given by $\partial q_{p l} / \partial t$. On the other hand the electrode temperature and with it the thermionic electron emission may be too low to supply at the beginning of the cathodic phase the arc current. In this case it has to be substituted by an enhanced ion current from the cathodic boundary layer being produced by an elevated cathode fall $U_{c}$.

For a more detailed discussion the cathode and the anode have to be considered 
separately. The current density in front of the cathode is composed of the density of a thermionically emitted current $j_{e m}\left(T_{\text {electrode }}\right)$, the density of an ion current $j_{i}\left(T_{e s}\right)$ and the density of an electron back diffusion current $j_{e p}\left(T_{e s}\right)$ from the plasma boundary layer to the cathode $[10]$ :

$$
j=j_{\text {em }}\left(T_{\text {electrode }}\right)+j_{i}\left(T_{e s}\right)-j_{e p}\left(T_{e s}\right)
$$

Taking the different contributions to the total current density into account the power flux density from the plasma boundary layer to the cathode can be written as:

$$
\begin{aligned}
q_{c} & =j_{i}\left(U_{i}-\Phi+U_{\text {kin }}-\frac{2 k_{B} T_{\text {electrode }}}{e}\right)+j_{e p} \frac{2 k_{B} T_{e s}}{e} \\
& +\alpha\left(T_{h}-T_{\text {electrode }}\right)-\left(j_{\text {em }}-j_{e p}\right)(\Phi-\Delta \Phi)-j_{e m} \frac{2 k_{B} T_{\text {electrode }}}{e}
\end{aligned}
$$

The product of $j_{i}$ and the first three terms in brackets gives the power flux density, which is transferred by the ions to the cathode. It is reduced by the enthalpy flux density of atoms, which balance the mass flow of ions to the cathode, being proportional to the fourth bracketed term. $e U_{i}$ is the ionisation energy of the filling gas, $e \Phi$ is the work function of the electrons, which neutralize the ions at the cathode surface, $e U_{k i n}$ is the kinetic energy of the ions and $T_{\text {electrode }}$ the local surface temperature of the electrode. The term proportional to $j_{e p}$ represents the enthalpy flux density which is transferred to the cathode by the electrons diffusing back from the plasma boundary layer to the cathode. The third term considers the heat transfer from the heavy particles in the boundary layer with the temperature $T_{h}$ in front of the electrode surface to the cathode. $\alpha$ is an empirical heat transfer coefficient [10]. The term $\left(j_{e m}-j_{e p}\right)(\Phi-\Delta \Phi)$ gives the power which is needed to lift the electrons from the conduction band within the electrode into free space reduced by the power which is released within the cathode by the back diffusing electrons. $\Delta \Phi$ is the Schottky reduction of the work function $\Phi$ by the electric field in front of the cathode. The last term considers the enthalpy flux density withdrawn from the cathode by the thermionic emission of electrons.

The expression for $q_{c}$ may be brought into a more appropriate form by the introduction of the total current density $j$ :

$$
\begin{aligned}
q_{c} & =-j \Phi+j_{i}\left(U_{i}+U_{k i n}-\frac{2 k_{B} T_{\text {electrode }}}{e}\right)+j_{\text {ep }} \frac{2 k_{B} T_{e s}}{e} \\
& -j_{\text {em }} \frac{2 k_{B} T_{\text {electrode }}}{e}+\alpha\left(T_{h}-T_{\text {electrode }}\right)+\left(j_{\text {em }}-j_{e p}\right) \Delta \Phi .
\end{aligned}
$$

If according to [10] the power balances of the electrons and of the heavy particles within the cathodic boundary layer and with them the cathode fall $U_{c}$ are taken into account the power flux density $q_{c}$ can be expressed by:

$$
q_{c}=j\left(U_{c}-\Phi-\frac{5}{2} \frac{k_{B} T_{e s}}{e}\right)-\frac{\partial q_{p l}}{\partial t}
$$

An integration of $q_{c}$ over the electrode surface gives the power balance of the electrode within the cathodic phase:

$$
P_{i n}=i\left(U_{c}-\Phi-\frac{5}{2} \frac{k_{B} \overline{T_{e s}}}{e}\right)-\frac{\partial Q_{c p l}}{\partial t}
$$


where $\overline{T_{e s}}$ is an average value of the electron temperature within the cathodic boundary layer and $Q_{c p l}$ the energy of the plasma boundary layer in front of the cathode.

The current density in front of the anode is composed of the same components as in front of the cathode, but with opposite sign and different amplitudes:

$$
j=j_{e p}\left(T_{e s}\right)-j_{e m}\left(T_{\text {electrode }}\right)-j_{i}\left(T_{e s}\right) .
$$

$j_{e p}$ is the dominant term in front of the anode. It is mainly supplied by the current from the bulk plasma.

The density of the power flux $q_{a}$ from the anodic plasma sheath to the anode surface can be written down straight forwardly $[16,19]$ :

$$
\begin{aligned}
q_{a} & =j_{e p}\left(\Phi+\frac{2 k_{B} T_{e s}}{e}\right)-j_{e m}\left(\Phi+\frac{2 k_{B} T_{a}}{e}\right) \\
& +j_{i}\left(U_{i}-\Phi+U_{k i n}-\frac{2 k_{B} T_{\text {electrode }}}{e}\right)+\alpha\left(T_{h}-T_{\text {electrode }}\right)
\end{aligned}
$$

The term proportional to $j_{e p}$ represents the flux of power which is released within the anode by the incident electrons. The product of $j_{\text {em }}\left(T_{\text {electrode }}\right)$ and the expression in brackets is the power flux density withdrawn from the anode by the thermionic emission of electrons. The term proportional to $j_{i}$ and the last term have the same meaning as in eq. 22. If $j_{e p}$ is substituted by the total current density $j$ according to eq. 26 then the power flux density $q_{a}$ can be written as:

$$
\begin{aligned}
q_{a} & =j\left(\Phi+\frac{2 k_{B} T_{e s}}{e}\right)+j_{e m}\left(\frac{2 k_{B} T_{e s}}{e}-\frac{2 k_{B} T_{\text {electrode }}}{e}\right) \\
& +j_{i}\left(U_{i}+U_{\text {kin }}+\frac{2 k_{B} T_{\text {es }}}{e}-\frac{2 k_{B} T_{\text {electrode }}}{e}\right)+\alpha\left(T_{h}-T_{\text {electrode }}\right)
\end{aligned}
$$

An integration of $q_{a}$ over the electrode surface yields the power input into the electrode within the anodic phase $[16,19]$ :

$$
\begin{aligned}
P_{\text {in }} & =i\left(\Phi+\frac{2 k_{B} \overline{T_{e s}}}{e}\right)+\int_{A} j_{e m}\left(\frac{2 k_{B} T_{e s}}{e}-\frac{2 k_{B} T_{\text {electrode }}}{e}\right) d A \\
& +\int_{A} j_{i}\left(U_{i}+U_{\text {kin }} \frac{2 k_{B} T_{e s}}{e}-\frac{2 k_{B} T_{\text {electrode }}}{e}\right) d A \\
& +\int_{A} \alpha\left(T_{h}-T_{\text {electrode }}\right) d A
\end{aligned}
$$

If the power balance of the whole boundary layer of a hot anode, consisting of the anodic sheath and the constriction zone in front of the anode, is considered a quite simple approximate expression for the power input $P_{\text {in }}$ into the anode can be given [19]:

$$
P_{\text {in }} \approx i\left(\Phi+\frac{5}{2} \frac{k_{B} \bar{T}_{e, a r c}}{e}\right)-\frac{\partial Q_{a p l}}{\partial t}
$$

$\bar{T}_{e, a r c}$ is an average value of the electron temperature within the arc plasma. The last term represents a power supply by the reduction of the energy content $Q_{\text {apl }}$ of the plasma 
sheath immediately in front of the anode.

An essential demand on HID electrodes in the case of switched dc or ac operation is a thermionic electron emission within the cathodic half period in the same order of magnitude as the total current. It can only be achieved if the cathode temperature is sufficiently high. As is shown by eq. 22 the power and temperature requirement within the cathodic phase represented by $j_{\text {em }}\left(T_{\text {electrode }}\right) \Phi$ is predominantly covered by the power flux transferred by the ion current density $j_{i}\left(T_{e s}\right)\left(U_{i}-\Phi+U_{k i n}-2 k_{B} T_{\text {electrode }} e^{-1}\right)$ [10], where

$$
\left(U_{i}-\Phi+U_{\text {kin }}-2 k_{B} T_{\text {electrode }} e^{-1}\right) \gg \Phi
$$

and therefore:

$$
j_{e m} \gg j_{i}
$$

It is supplemented by a minor contribution transferred by the density of the electron back diffusion current $j_{e p}$ and by a heat transfer from the heavy particles within the plasma to the cathode. The requirement is met by an adjustment of the cathode fall $U_{c}$ according to eq. 24 and eq. 25.

\subsection{Operation with a switched dc current}

The measurements of $T_{\text {tip }}$ and $P_{\text {in }}$ for switched dc currents, given in figure 3 , figure 4, figure 5 , and table 1 show, that both quantities deviate within the cathodic phase only marginally from the corresponding values of the cathode operated with a dc current, but in part considerably from those of a dc anode. From this finding the conclusion can be drawn, that the electrode temperature is mainly controlled by the temperature requirement for thermionic electron emission within the cathodic phase if the electrode is operated with a switched dc current. At the beginning of the anodic phase the electrode temperature $T_{t i p}$ and the power input $P_{\text {in }}$ start to decrease for low currents and to increase for higher currents $(i \geq 4.8 \mathrm{~A}$ for $f=10 \mathrm{~Hz})$ in the case of a switched dc operation as is shown in figure 3 and figure 4 . The cooling down within the anodic phase at low currents is compensated by an enhanced power input at the beginning of the cathodic phase. It is clearly demonstrated by the commutation peaks which are shown by the courses of $\operatorname{ESV}(\varphi)$ after current zero crossing, e. g. in figure 6 . They are produced by an enhanced cathode fall $U_{c}$, which raises by an increase of the electron temperature and charged particle density within the cathodic boundary layer the ion current towards the cathode and with it according to eq. 22 the power input into the cathode. But with increasing operating frequency the time interval and with it the amplitude of the temperature drop and the variation of the energy input decreases and accordingly the amplitude of the commutation peak. It vanishes if the electrode temperature within the anodic phase is equal or even higher than within the cathodic phase.

By reason of the high starting point of the electrode temperature at the change over from the cathodic to the anodic phase the thermionic electron emission remains high within 
the anodic phase. It produces according to eq. 20, eq. 28 and eq. 29 an additional power transfer to the anode, which raises the anode temperature $T_{\text {electrode }}$ and lowers the electron temperature $T_{e s}$ within the sheath in front of the anode [16, 19]. Moreover high values of $j_{e m}$ within the anodic phase are accompanied by a decrease of $j_{i}$ in eq. 28 and 29 [19].

The power transfer during the anodic phase by thermionic electron emission can be estimated, if it is assumed that by reason of the nearly equal electrode temperature the thermal electron emission is in the same order of magnitude as within the cathodic phase. It is approximately given by the current amplitude $i$. For $i=3 \mathrm{~A}, f=10 \mathrm{~Hz}$ additionally the electron temperature $T_{e s}$ within the plasma sheath in front of the electrode is available [33]. It is approximately $\overline{T_{e s}} \approx 7600 \mathrm{~K}$ within the anodic phase. The electrode tip temperature amounts to $T_{\text {tip }} \approx 3140 \mathrm{~K}$ according to figure $3 \mathrm{a}$. The estimate of the additional power input into the anodic phase by thermionically emitted electrons gives:

$$
\int_{A} j_{e m}\left(\frac{2 k_{B} \overline{T_{e s}}}{e}-\frac{2 k_{B} T_{\text {electrode }}}{e}\right) d A \approx i\left(\frac{2 k_{B} \overline{T_{e s}}}{e}-\frac{2 k_{B} T_{\text {tip }}}{e}\right)=2.3 \mathrm{~W}
$$

It makes only a minor contribution to the total power input into the electrode.

An overlap may occur of the electrode temperature increase and of the plasma temperature reduction from the anodic onto the cathodic phase, growing with frequency. It seems to be responsible for the weak rise of the phase averaged values of $T_{\text {tip }}$ and $P_{\text {in }}$ with increasing operating frequency. It may be caused in part by the increased power input into the electrode within the anodic phase with increasing electrode temperature and in part by the reduced electron temperature $\overline{T_{e s}}$ in front of the electrode during the cathodic phase accompanied by a reduction of the electron enthalpy flux from the boundary layer into the bulk plasma according to eq. 25. The rising is widely completed if the electrode temperature within the anodic phase has adapted to the electrode temperature within the cathodic phase. Further investigations are needed to achieve a correct interpretation of the elevation of $T_{t i p}$ and $P_{i n}$ with increasing operating frequency.

If the electrode is heated during the anodic phase the electrode temperature seems to approach at higher frequencies a tip temperature, which is lower than the arithmetic average of the tip temperature of a dc cathode and dc anode operated with the same current amplitude. But when the electrode temperature declines within the anodic phase the temperature $T_{\text {tip }}$ approaches at higher frequencies a value, which is higher than the arithmetic average of the tip temperature of the corresponding dc cathode and dc anode. The dependence of the arithmetic average of $T_{t i p}$ on current and frequency demonstrates the decreasing influence of the anodic phase on the electrode temperature with increasing operation frequency. 


\subsection{Operation with a sinusoidal current}

The variation of $T_{t i p}$ and $P_{i n}$ in dependence on the phase angle $\varphi$ becomes more complicated in the case of an operation with a sinusoidal current. But some general aspects remain unchanged. Especially the demand on a sufficiently high electrode temperature for thermionic electron emission within the cathodic phase is still effective. It determines the average electrode temperature which is only modified to some extent by the power input into the anodic phase. For the special conditions in figure 7 and figure 8 it is lower than the power input into the cathodic phase below a current of $i_{r m s}=7.2 \mathrm{~A}$ for $f=10 \mathrm{~Hz}$, which is much higher than the corresponding current in the case of a switched dc operation. The lower power input within the anodic phase is compensated as in the case of an operation with a switched dc current by an enhanced power input at the beginning of the cathodic phase as is indicated by the exaggerated ESV values after current zero crossing being shown in figure 10. The input power within the anodic phase approaches that within the cathodic phase with increasing current amplitude and/or operating frequency. Simultaneously the course of $E S V(\varphi)$ becomes more and more sinusoidal. The interaction between the plasma boundary layer and the electrode by a surplus of thermionic electron emission becomes more intense than in the case of a switched dc operation. It will be effective not only within the anodic phase but also within the cathodic phase after current maximum. The differences between an operation with a switched dc current and a sinusoidal current, represented by $T_{\text {tip }}$ and $P_{\text {in }}$, disappear for operating frequencies of several $\mathrm{kHz}$.

The measurements given in figure 8 show that at current zero crossing

$$
P_{i n}(i=0)>0 \text {. }
$$

This result needs a special consideration since it may be not only a result of measuring errors mainly caused by an insufficient phase resolution.

For $j=0$ the relation of the current densities in front of the electrode become according to eq. 21 and eq. 26 :

$$
j_{e p}=j_{e m}+j_{i}
$$

$j_{i}$ may be neglected compared to $j_{e m}$ in the vicinity of current zero crossing as the consequence of a low electron temperature in front of the electrode:

$$
j_{e p} \approx j_{e m}
$$

Approaching current zero from the cathodic side the density of the power input $P_{\text {in }}$ from the plasma to the electrode can be written as according to eq. 23 and eq. 24:

$$
\begin{aligned}
P_{\text {in }}(i=0) & =\int_{A} j_{e m}\left(\frac{2 k_{B} T_{\text {es }}}{e}-\frac{2 k_{B} T_{\text {electrode }}}{e}\right) d A \\
& +\int_{A} \alpha\left(T_{h}-T_{\text {electrode }}\right) d A=-\frac{\partial Q_{c p l}}{\partial t}
\end{aligned}
$$

Approaching current zero from the anodic side the same result is found from eq. 29 with $Q_{c p l}=Q_{a p l}$ for $i=0$. 
An upper limit of the power transfer by thermionic electron emission $P_{e m}$ represented by the first term in eq. 37 can be estimated. For $i_{r m s}=3 \mathrm{~A}, f=50 \mathrm{~Hz}$ a measurement of $T_{e s}$ at current zero crossing is available [33]. It is in the order $\overline{T_{e s}} \approx 5000 \mathrm{~K}$ at intervals of $z=62.5 \mu \mathrm{m}$. It may be even lower at smaller distances from the electrode. The electrode temperature amounts to $T_{\text {electrode }} \approx 3150 \mathrm{~K}$ according to figure 7 . The current

$$
I_{\text {em }}=\int_{A} j_{\text {em }}\left(T_{\text {electrode }}\right) d A,
$$

generated by thermionic electron emission at current zero crossing is in each case less than the current maximum within the cathodic phase:

$$
I_{e m}<\hat{i}=4.24 \mathrm{~A}
$$

Inserting these numbers it is found:

$$
P_{e m} \approx I_{e m} 2 k_{B} e^{-1}\left(T_{e s}-T_{\text {electrode }}\right) \approx 1.35 \mathrm{~W} .
$$

To make an estimate of the second term in eq. 35 appropriate values of $T_{h}$ and $\alpha$ are needed. At current zero crossing the electric field strength within the plasma boundary layer in front of the electrode is $E=0$ resulting in an adjustment of the electron temperature to the gas temperature [39, 40]: $T_{h} \leq T_{e s} \leq 5000 \mathrm{~K}$. A good approximation of the experimental results is achieved if $\alpha=5000 \mathrm{Wm}^{-2} \mathrm{~K}^{-1}$ is used in simulations of the cathodic arc attachment $[41,42]$. The power $P_{h}$ transferred by heat conduction from the plasma to the electrode is mainly absorbed by the end face of the electrode $r^{2} \pi=0.785 \cdot 10^{-6} \mathrm{~m}^{2}$. Taking into account these values yields:

$$
\begin{aligned}
& P_{h}=r^{2} \pi \alpha\left(T_{h}-T_{\text {electrode }}\right) \leq 7.3 \mathrm{~W} \\
& P_{\text {em }}+P_{h} \leq 8.65 \mathrm{~W}<P_{\text {in }}(i=0) \approx 18.5 \mathrm{~W}
\end{aligned}
$$

The measuring value $P_{\text {in }}(i=0)$ is taken from figure 8 a. The discrepancy between the measured and estimated power input is presumably mainly caused by an insufficient phase resolution of the measurement in the vicinity of current zero crossing and only in part by a too rough estimate. To clarify this point temperature measurements with higher phase resolution in the vicinity of current zero crossing are required. They may offer the chance to get more information about the power transfer to the electrode by heat conduction.

The estimated value of $P_{e m} \approx 1.35 \mathrm{~W}$ is in the same order of magnitude as the power input being estimated in eq. 33. It corresponds to the rise of the input power $P_{\text {in }}$ when the operating frequency is increased from $10 \mathrm{~Hz}$ to $10 \mathrm{kHz}$. It indicates that it is caused at least in part by thermionic electron emission within the anodic half period.

\subsection{Comparison of the power input within the cathodic and anodic phase}

A generally equal power input within the cathodic and anodic phase at higher operating frequencies, suggested by figure 4 and figure 8 , seems to be doubtful since the 
consequence would be according to eq. 25 and eq. 30 a cathode fall, which is independent of the electrode geometry at higher operating frequencies.

$$
U_{c} \approx 2 \phi+\frac{5}{2} \frac{k_{B}\left(\bar{T}_{e, a r c}+\bar{T}_{e s}\right)}{e}+\left[\frac{1}{i}\left(\frac{\partial Q_{c p l}}{\partial t}-\frac{\partial Q_{a p l}}{\partial t}\right)\right]
$$

The term in square brackets can be generally neglected in some distance from current zero crossing.

The general validity of this far-reaching statement needs a more detailed investigation. For an electrode with the same dimensions operated with a dc current of $I=3.6 \mathrm{~A}$, at which the power input into the cathode and anode becomes equal, the measured cathode fall [11] and the cathode fall from eq. $43[12]$ amount to $U_{c}=14.3 \mathrm{~V}$ and $U_{c}=13.7 \mathrm{~V}$, respectively. It is in agreement within the limit of error indicating, that eq. 43 really applies, if the power input into the cathode and anode is equal.

\section{Conclusions}

By varying the operating frequency of the model lamp between $10 \mathrm{~Hz}$ and $10 \mathrm{kHz}$ a transition region between two different operation modes is realized. At low operating frequencies distinct differences between the cathodic and anodic phase exist, which reflect the different properties of the cathodic and anodic arc attachment being operated with a dc current. But at frequencies of some $\mathrm{kHz}$ the differences between the electrode tip temperature within the cathodic and anodic phase have nearly disappeared. The electrode temperature is mainly determined by the thermionic electron emission, by which the current transfer within the cathodic phase is realized, and the extension of the arc attachment zone on the electrode surface mainly by the interaction between the arc plasma and the electrode by thermionic electron emission of the electrode within the anodic phase. The electrode tip temperature differs only marginally from that of a dc cathode operated with the same rms current. There is no indication for a noteworthy contribution of a displacement current to the current transfer between plasma and electrode at $10 \mathrm{kHz}$. The findings may be an appropriate starting point to simulate the operation of HID electrodes with a switched dc current of several $\mathrm{kHz}$.

A weak increase of the electrode tip temperature and of the power input is found with increasing operating frequency if the lamp is operated with an argon filling between electrodes made of pure tungsten. It may be caused by an additional heating of the anode by thermionic electron emission and a reduced electron enthalpy flux from the cathodic boundary layer into the bulk plasma.

When a reduction of the work function by an emitter effect comes into operation at a de cathode but not at the corresponding dc anode the situation may be different. In this case the emitter effect is produced by a monolayer of electropositive atoms, which may be sustained by an ion current from the plasma to the cathode. At higher frequencies this layer may survive also during the anodic phase and with it the reduction of the work function. As a consequence at higher frequencies not only the power input within the cathodic phase is reduced but also within the anodic phase [33]. More details 
about pyrometric and spectroscopic measurements in a YAG-lamp with a $\mathrm{Hg} / \mathrm{DyI}_{3^{-}}$ filling $[26,27]$ will be given in a subsequent paper. Preliminary results are already given in $[45]$.

A cathode spot is characterized by a low global electrode temperature but a high temperature within the attachment zone of the arc $[8,11]$. The plasma jet, which is emitted by the attachment zone of the spot towards the tube wall, may disturb lamp operation. The probability that a spot mode arises becomes high if a commutation peak after current zero crossing is formed [21]. During the formation of a spot the local current density may increase exponentially according to $\exp (\Omega t)$. The transition from the diffuse to the spot mode takes place with a low growth rate $\Omega$, especially if the electrode temperature is high and the cathode fall low. $\Omega$ is in the order of $1000 \mathrm{~s}^{-1}[21]$. The probability for the formation of a cathode spot decreases with increasing operating frequency by reason of the short cathodic half period, high electrode temperature and diffuse arc attachment within the anodic phase. For an operating frequency of several $\mathrm{kHz}$ it becomes quite low.

An arc attachment at the electrode by an asymmetric spot may induce a helical magnetic instability of the arc column $[43,44]$. Therefore the stability of the discharge will be improved with increasing operating frequency as long as the frequency is below the lowest acoustic resonance frequency of the lamp tube.

The investigation shows that it may be favourable to run HID lamps with operating frequencies of some $\mathrm{kHz}$ especially if in addition an appropriate emitter effect can be used.

\section{Acknowledgements}

Financial support from Deutsche Forschungsgemeinschaft (Graduiertenkolleg 1051) and from the Research School of the Ruhr-University of Bochum is gratefully acknowledged by the authors.

\section{References}

[1] Riemann K U 1991 J. Phys. D: Appl. Phys. 24 493-518

[2] Benilov M S and Marotta A 1995 J. Phys. D: Appl. Phys. 28 1869-82

[3] Schmitz H and Riemann K U 2002 J. Phys. D: Appl. Phys. 35 1727-1735

[4] Benilov M S and Cunha MD 2002 J. Phys. D: Appl. Phys. 35 1736-50

[5] Dabringhausen L et al 2002 J. Phys. D: Appl. Phys. 35 1621-1630

[6] Luhmann J et al 2002 J. Phys. D: Appl. Phys. 35 1631-1638

[7] Nandelstaedt D et al 2002 J. Phys. D: Appl. Phys. 35 1639-1647

[8] Lichtenberg S et al 2002 J. Phys. D: Appl. Phys. 35 1648-1656

[9] Hartmann T et al 2002 J. Phys. D: Appl. Phys. 35 1657-1667

[10] Lichtenberg S et al 2005 J. Phys. D: Appl. Phys. 38 3112-3127

[11] Dabringhausen L et al 2005 J. Phys. D: Appl. Phys. 38 3128-3142

[12] Redwitz M et al 2005 J. Phys. D: Appl. Phys. 38 3143-3154

[13] Benilov M S, Carpaij M and Cunha MD 2006 J. Phys. D: Appl. Phys. 39 2124-34

[14] Benilov M S 2008 J. Phys. D: Appl. Phys. 41144001 
[15] Scharf F H and Brinkmann R P 2008 J. Phys. D: Appl. Phys. 41185206

[16] Redwitz M et al 2006 J. Phys. D: Appl. Phys. 39 2160-2179

[17] Almeida N A et al 2009 J. Phys. D: Appl. Phys. 42045210

[18] Heberlein J et al 2010 J. Phys. D: Appl. Phys. 43023001

[19] Mentel J and Heberlein J 2010 J. Phys. D: Appl. Phys. 43023002

[20] Hartmann T et al 2010 J. Phys. D: Appl. Phys. 43025201

[21] Langenscheidt O et al 2007 J. Phys. D: Appl. Phys. 40 415-431

[22] Reinelt J et al 2008 J. Phys. D: Appl. Phys. 41144002

[23] Kloss A et al $2000 \mathrm{~J}$. Appl. Phys. 88 1271-5

[24] Kloss A et al 2001 Appl. Phys. Lett. 78 3027-8

[25] Fromm DC et al 2002 J. Phys. D: Appl. Phys. 35 1668-80

[26] Luijks G M J F et al 2005 J. Phys. D: Appl. Phys. 38 3163-3169

[27] Langenscheidt O et al 2008 J. Phys. D: Appl. Phys. 41144005

[28] Luijks G M J F et al 2008 J. Phys. D: Appl. Phys. 41144006

[29] Westermeier M et al 2008 IEEE Transactions on Plasma Science 36 (4) 1176-1177

[30] Boetticher R and Boetticher W 2001 J. Phys. D: Appl. Phys. 34 1110-15

[31] Pursch H et al 2002 J. Phys. D: Appl. Phys. 35 1757-60

[32] Lenef A I 2008 J. Phys. D: Appl. Phys. 41144003

[33] Reinelt J 2009 Experimental investigation on electrodes of HID lamps at low and high operating frequencies PhD thesis Ruhr University Bochum

[34] DeVos J C 1954 Physica 20 690-714

[35] White G K and Minges M L 1997 Int. J. Thermophys. 1269-327

[36] Yih S W H and Wang C T 1979 Tungsten: Sources, Metallurgy, Properties and Application (New York: Plenum)

[37] Gustafson P 1985 Int. J. Thermophys. 6 395-409

[38] Dabringhausen L 2004 Charakterisierung von Elektroden für Hochdruck-Plasmalampen durch Pyrometrie und Simulation PhD thesis (Berlin Tenea) Ruhr Universität Bochum

[39] Kuehn G and Kock M 2006 J. Phys. D: Appl. Phys. 39 2401-14

[40] Kuehn G and Kock M 2007 Physical Review E 75016406

[41] Scharf F H et al 2007 Proc. Int. Conf. on Phen. Ion. Gases (ICPIG) XXVIII 142

[42] Langenscheidt O 2008 Elektroden fuer HID-Lampen - Diagnostik und Simulation PhD thesis Ruhr University Bochum

[43] Huelsmann H G and Mentel J 1987 Phys. Fluids 30 2266-73

[44] Huelsmann H G and Mentel J 1987 Phys. Fluids 30 2274-78

[45] Reinelt J et al 2009 Investigation of the anodic and cathodic emitter-effect in high-intensitydischarge (HID) lamps Proc. 19th International Symposium on Plasma Chemistry (Bochum, 27-31 July 2009) ed von Keudell A et al P.1.1.14 